Research Article

\title{
Hybrid OFDMA Resource Allocation Scheme for Ensuring Required Level of Proportional Fairness
}

\author{
Ming Sun $\mathbb{D}^{1},{ }^{1}$ Kangle Zhai, ${ }^{1}$ Wei Cao, ${ }^{1}$ Ying Wang, ${ }^{1}$ and Yaoqun $\mathrm{Xu}^{2}$ \\ ${ }^{1}$ College of Computer and Control Engineering, Qiqihar University, Qiqihar 161006, China \\ ${ }^{2}$ School of Computer and Information Engineering, Harbin University of Commerce, Harbin 150028, China \\ Correspondence should be addressed to Ming Sun; snogisunming@163.com
}

Received 6 February 2020; Revised 17 August 2020; Accepted 10 October 2020; Published 23 October 2020

Academic Editor: David Greiner

Copyright (c) 2020 Ming Sun et al. This is an open access article distributed under the Creative Commons Attribution License, which permits unrestricted use, distribution, and reproduction in any medium, provided the original work is properly cited.

The new-generation wireless communication networks are envisioned to offer higher sum data rates along with the required level of fairness. Previous works tend to suffer from a decayed performance as subcarriers become relatively insufficient in allocation to users. To maximize the sum data rates and ensure the required level of proportional fairness, this paper presents a hybrid OFDMA resource allocation scheme which uses Hungarian algorithm combined with a greedy method for subcarrier allocation and uses bee colony optimization for power allocation. The proposed subcarrier allocation scheme can make full use of advantages of both globally optimal Hungarian algorithm in enhancing sum data rates and locally optimal greedy method in maintaining a reasonable fairness level and can make Hungarian algorithm work in a searching mode for further improvement of sum data rates and fairness. The proposed power allocation scheme can converge to the required level of proportional fairness but with higher sum data rates if the subcarrier allocation does not achieve the required fairness. Simulation results show that the proposed scheme can obtain the required level of proportional fairness but with higher sum data rates even if subcarriers are relatively insufficient in allocation to users. Complexity analysis shows the proposed method has moderate complexity.

\section{Introduction}

Huge requirements of users for higher data rates along with quality of services (QoS) have led the wireless communication networks to be transformed from $4 \mathrm{G}$ to $5 \mathrm{G}$. Orthogonal frequency division multiple access (OFDMA), which is efficient in spectrum utilization and robust in channel fading, has been a successful multiple access policy to provide high data rates in 4G. By taking into account of backward compatibility of orthogonal frequency division multiplexing (OFDM) with the existing technologies in $4 \mathrm{G}$ as well as the advantages mentioned above, OFDM is also considered as a candidate for $5 \mathrm{G}$ by certain enhancements [1-4]. From this viewpoint, it is worthwhile to further improve the data rates with QoS for OFDMA systems.

Resource allocation involved in OFDMA, nonorthogonal multiple access (NOMA), and rate splitting multiple access (RSMA) have been an active research aspect in wireless communication systems, and various techniques such as greedy algorithms, evolutionary algorithms, and artificial intelligent algorithms have been applied for resource allocation [5-31]. In the downlink of an OFDMA net cell, rate adaptive optimization is to maximize the sum data rate transmitted to users by a base station with the constraint of the total transmit power of the base station [5], and the resource allocation is referred to as the base station allocation of subcarriers and power to users. Joint subcarrier and power allocation, i.e., simultaneous optimization of subcarrier and power, tend to be computationally intensive because of its NP-hard nature $[6,7]$. As a result, separate subcarrier and power allocation or even subcarrier allocation with equal power allocation is more likely to be used because of the lower computational complexity [5].

As one of the QoS indices, the proportional fairness among users, which is predetermined by a proportional data rate constraint [5], can be used to ensure each user to obtain the required data rate. Hence, maximizing the sum data rate and simultaneously ensuring the proportional fairness 
among users draw extensive and increasing attention persistently. However, the maximization of the sum data rate conflicts with the proportional data rate fairness by nature. That is, a higher proportional fairness easily leads to a lower sum data rate.

Many algorithms focus on balancing the tradeoff between the sum data rate and the proportional data rate fairness. For example, a greedy based method was proposed in [13] where the user with the lowest proportional data rate has priority to select the subcarrier with the highest channelto-noise ratio. Evolutionary algorithms including ant colony algorithm [4, 14], bee colony algorithm [15], and genetic algorithm [16], were applied for rate adaptive resource allocation with proportional fairness. Those evolutionary algorithms can obtain higher sum data rates and hold the predetermined proportional data rate constraint well as subcarriers are relatively sufficient for users. However, as far as our experimental verification, evolutionary algorithms easily suffer from a seriously decayed performance as subcarriers become relatively insufficient in allocation to users.

By introducing fairness threshold mechanism into the proportional data rate constraint, the algorithm in $[17,18]$ used a greedy method of subcarrier allocation along with a particle swarm optimization- (PSO-) based power allocation to enhance the sum data rate and satisfy the lowest fairness flexibly. However, because of the greedy characteristics in maximizing the sum data rate, the greedy method is probably not to achieve the predetermined fairness threshold in subcarrier allocation even if subcarriers are relatively sufficient in allocation to users. Consequently, in order to meet the predetermined fairness threshold, the PSO-based power allocation would be used inevitably and frequently, thereby resulting in inefficiency to maximize the sum data rates due to the premature convergence of the PSO $[17,18]$.

Instead of the proportionality of data rates, both the algorithms in $[19,20]$ used the proportionality of the number of subcarriers as the constraint, thereby obtaining acceptable proportional fairness while higher sum data rate than the algorithm in [13]. However, both of them are completely based on greedy methods with local optimization. Hence, it is requisite to improve them by global optimization to meet incremental requirements of large numbers of users for higher data rates along with more reasonable proportional fairness.

From the above reviews, the previous works tend to suffer from a decayed performance as subcarriers become relatively insufficient in allocation to users. However, the new-generation wireless communication networks are envisioned to offer higher sum data rates along with the required level of fairness. So these conventional methods in the references mentioned above cannot be applied in this paper. In this paper, to maximize the sum data rates and ensure the required level of proportional fairness, we propose a hybrid OFDMA resource allocation scheme which uses globally optimal Hungarian algorithm combined with the greedy method similar to [19] for subcarrier allocation and uses bee colony optimization for power allocation. Our work is different from the previous works in the following three aspects. Firstly, advantages of both globally optimal Hungarian algorithm and locally optimal greedy method are effectively combined to enhance the sum data rate and maintain a reasonable fairness level in subcarrier allocation. Secondly, Hungarian algorithm can work in a searching mode to obtain better solutions with both higher sum data rate and higher level of fairness in subcarrier allocation. Thirdly, bee colony optimization can be used to ensure the required level of proportional fairness and maximize the sum data rates, and it is used only if the fairness obtained in subcarrier allocation is lower than the required fairness.

The rest of this paper is structured as follows. Section 2 provides the formulation of rate adaptive resource allocation with the proportional data rate fairness. Subcarrier allocation and power allocation of the proposed hybrid scheme are described in detail, respectively, in Sections 3 and 4. Section 5 presents the simulation results and complexity analysis, and in the final, Section 6 concludes this paper.

\section{System Model}

The OFDMA system model is shown in Figure 1. The system has one base station that transmits through a slowly timevarying and Rayleigh fading channel with a bandwidth $B$. Besides, the channel is divided into $N$ frequency orthogonal subcarriers shared by $K$ users in an OFDMA system, and the total transmit power of the base station is $P_{T}$. Finally, the channel state information is assumed to be completely known to the base station, and the channel is assumed to be unchanged during resource allocation.

The formulation of rate adaptive resource allocation with the required level of proportional fairness is shown as follows:

$$
\max _{p_{k, n}, c_{k, n}} \sum_{k=1}^{K} \sum_{n=1}^{N} \frac{B}{N} c_{k, n} \log _{2}\left(1+p_{k, n} G_{k, n}\right),
$$

subject to the following constraints:

$$
\begin{gathered}
\sum_{k=1}^{K} c_{k, n}=1, \quad c_{k, n} \in\{0,1\}, \forall k, n, \\
\sum_{k=1}^{K} \sum_{n=1}^{N} c_{k, n} p_{k, n} \leq P_{T}, \quad p_{k, n} \geq 0, \forall k, n, \\
F=\frac{\left(\sum_{k=1}^{K}\left(R_{k} / \lambda_{k}\right)\right)^{2}}{K \sum_{k=1}^{K}\left(R_{k} / \lambda_{k}\right)^{2}} \geq \varepsilon .
\end{gathered}
$$

Equation (1) represents the optimization objective of rate adaptive resource allocation problem. Equation (2) is the constraint that each subcarrier is uniquely allocated to a single user. Equation (3) shows that the sum of the assigned transmit powers should be less than or equal to the total transmit power. Equation (4) represents the constraint of the required level of proportional fairness.

In (1)-(4), $G_{k, n}=\left|h_{k, n}\right|^{2} /\left(N_{0} B / N\right), h_{k, n}$ is the subcarrier gain of user $k$ in subcarrier $n ; N_{0}$ is the noise power density; $c_{k, n}$ represents whether subcarrier $n$ is assigned to user $k ; c_{k, n}$ 


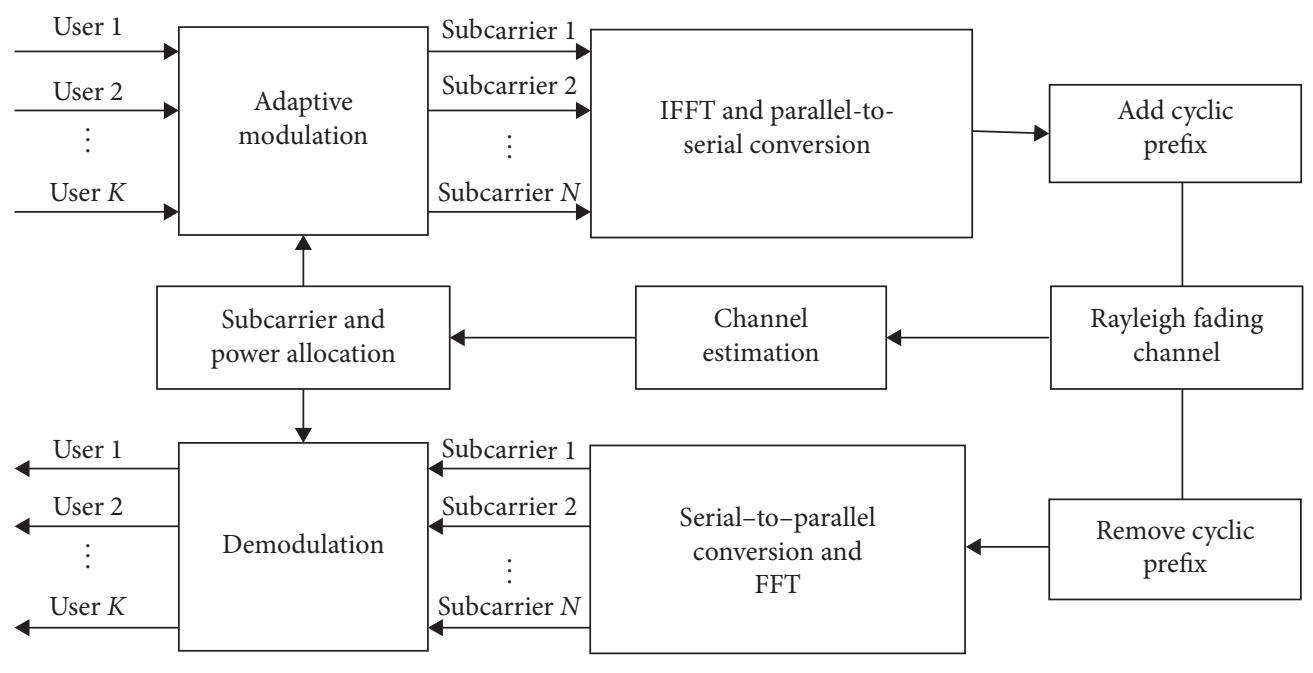

Figure 1: OFDMA system model.

is 1 if subcarrier $n$ is assigned to user $k$; otherwise, $c_{k, n}$ is 0 ; $p_{k, n}$ is the assigned power of user $k$ in subcarrier $n$, greater than or equal to zero; $R_{k}$ is the data rate of user $k$, shown below:

$$
R_{k}=\sum_{n=1}^{N} \frac{B}{N} c_{k, n} \log _{2}\left(1+p_{k, n} G_{k, n}\right) .
$$

where $F$ is the index of the proportional data rate fairness $[5,7,15] ; \varepsilon$ is the required level of proportional fairness; and $\lambda_{k}$ is the predetermined data rate proportionality value of user $k$. The index $F$ varies from 0 to 1 . Note that a larger index $F$ indicates a better proportional fairness among users. The index $F$ will be equal to 1 if the following equation comes into existence:

$$
R_{1}: R_{2}: \cdots: R_{K}=\lambda_{1}: \lambda_{2}: \cdots: \lambda_{K}
$$

\section{Related Work}

In the following, we present reviews on both subcarrier allocation based on Hungarian algorithm and power allocation for ensuring proportional fairness which are relevant to our proposed work.

\subsection{Subcarrier Allocation Based on Hungarian Algorithm.} Owing to the global optimization ability, Hungarian algorithm has been used for rate adaptive resource allocation [21-23]. For example, the Hungarian method in [21] was used for subcarrier allocation in downlink of an OFDMAbased multicell underlay cognitive radio network. In [22], the Hungarian method was used for joint allocation of subcarrier and power by relaxing constraints. Nevertheless, Hungarian algorithm in $[21,22]$ is only applied to maximize the sum data rate while not used for the fairness. Hungarian algorithm was used to deal with the fairness in [23], where the remaining $N-K$ subcarriers are, respectively, allocated to users by running Hungarian algorithm once for each subcarrier. However, this undoubtedly leads to a sharp increase in amount of computation because the value of $N-K$ is large in general.

3.2. Power Allocation for Ensuring Proportional Fairness. PSO has been used in power allocation to achieve the predetermined fairness threshold in $[17,18]$ wherein PSO is combined with a criterion presented in [25] to deal with the constraints. The criterion for handling the constraints can be described as follows: (1) a feasible solution is superior to an unfeasible solution; (2) unfeasible solutions are compared based on their constraint violations: an unfeasible solution with smaller constraint violation is better than the one with larger constraint violation; (3) feasible solutions are compared based on their fitness: a feasible solutions with larger fitness outperforms the one with smaller fitness.

However, a basic PSO tends to have more parameters than a basic bee colony algorithm. Apart from the common parameters such as maximum number of cycles and lower and upper bound of variables, the basic PSO has four parameters including inertia weight, social constant, cognition constant, and limit values for velocities, while the basic bee colony algorithm has only one parameter of the predetermined number of cycles to abandon food sources This implies that PSO is more troublesome than bee colony algorithm in parameter tuning. On the other hand, bee colony algorithm has been proven to be superior to PSO on a large amount of unconstrained test functions $[15,24]$.

Artificial bee colony algorithm (ABC) with the fitness function shown in (7) has been used for the proportional data rate fairness in power allocation [26, 27]:

$$
\text { fitness }=F \sum_{k=1}^{K} R_{k} \text {. }
$$

With the help of the fitness function (7), the ABC in $[26,27]$ is more likely to obtain higher sum data rate. This is because that the sum data rate has more impact on the fitness function (7) than the index of the proportional fairness $F$. As a result, the $\mathrm{ABC}$ with the fitness function (7) would not 
achieve the required level of proportional fairness if subcarriers become relatively insufficient in allocation to users.

Based on a principle that $\left(p_{k, n}+1 / G_{k, n}\right)$ equals to $\left(p_{k, m}+1 / G_{k, m}\right)$ as $n \neq m$, the strict proportional fairness (i.e., $F=1$ ) can be achieved by a power allocation algorithm in [5]. However, in order for the strict proportional fairness, numerical algorithms like the Newton-Raphson method should be applied to solve a complex nonlinear equation. Because of conflicts between sum data rate and proportional fairness, the strict proportional fairness undoubtedly leads to a much lower sum data rate, which cannot meet the requirement of users for higher sum data rates.

Because of shortcomings of the previous works on both subcarrier allocation and power allocation, the conventional methods mentioned above cannot be applied for the rate adaptive resource allocation problem (1)-(4) in this paper. Motivated from this, we propose a hybrid OFDMA resource allocation scheme which uses globally optimal Hungarian algorithm combined with a greedy method for subcarrier allocation to enhance the sum data rate and hold a reasonable fairness level and uses bee colony optimization for power allocation to ensure proportional fairness and maximize the sum data rates.

\section{Proposed Hybrid OFDMA Resource Allocation Scheme}

In our proposed hybrid OFDMA resource allocation scheme, subcarrier allocation and power allocation are adopted, respectively, for solving the rate adaptive resource allocation with the required level of proportional fairness (1)-(6), as follows.

Subcarrier allocation: the policy of equal power allocation is adopted in this paper, i.e., $p=P_{T} / N$. Then, the model of subcarrier allocation can be formulated as follows:

$$
\max _{c_{k, n}} \sum_{k=1}^{K} \sum_{n=1}^{N} c_{k, n} \log _{2}\left(1+p G_{k, n}\right)
$$

subject to the constraints of (2) and (6).

Power allocation: because $c_{k, n}$ is fixed in subcarrier allocation, the model of power allocation can be formulated as follows:

$$
\max _{p_{k, n}} \sum_{k=1}^{K} \sum_{n=1}^{N} c_{k, n} \log _{2}\left(1+p_{k, n} G_{k, n}\right)
$$

subject to the constraints of (3) and (4).

\subsection{Proposed Hungarian Algorithm Combined with Greedy} Method for Subcarrier Allocation. Using the proportionality of the number of subcarriers approximate to the proportionality of data rates, the greedy method in [19] can roughly satisfy the proportionality constraint and obtain higher sum data rate. However, the greedy method can be further improved by the globally optimal algorithms. In this paper, we propose to combine Hungarian algorithm with the greedy method [19] and can further make Hungarian algorithm work in a searching mode.

The proportionality of the number of subcarriers is first set to be approximate to the data rate proportionality, i.e., $N_{1}: N_{2}: \cdots: N_{K} \approx \lambda_{1}: \lambda_{2}: \cdots: \lambda_{K}$, where $N_{k}$ is the number of subcarriers assigned to user $k$, formulated as

$$
N_{k}=\left\lfloor\frac{N \lambda_{k}}{\sum_{i=1}^{K} \lambda_{i}}\right\rfloor .
$$

Besides, $N^{*}$ is defined as

$$
N^{*}=N-\sum_{k=1}^{K} N_{k} \text {. }
$$

Based on the predetermined proportionality of the number of subcarriers, the proposed subcarrier allocation with equal power allocation is described as follows:

Step 1: initialize $c_{k, n}=0, R_{k}=0, T_{\mathrm{op}}=0, F_{\mathrm{op}}=0, N_{\mathrm{op}}=0$, $F^{*}=0, p=P_{T} / N, \Omega=\{1,2, \ldots, N\}$ and calculate $N_{k}$ and $N^{*}$ according to (10) and (11) for $\forall k \in\{1,2, \ldots, K\}$ and $\forall n \in\{1,2, \ldots, N\}$. Determine whether Hungarian algorithm works in the searching mode.

Step 2: as the value of $k$ varies from 1 to $K$, perform

$$
\begin{aligned}
& n=\operatorname{argmax}_{n \in \Omega} G_{k, n} \\
& c_{k, n}=1, N_{k}=N_{k}-1, \Omega=\Omega-\{n\} \\
& R_{k}=R_{k}+(B / N) \log _{2}\left(1+p G_{k, n}\right)
\end{aligned}
$$

Step 3: set $N_{\text {rest }}=N^{*}$ and $\Omega^{*}=\Omega$

Step 4: set $\Phi=\{1,2, \ldots, K\}$, and perform

$$
\begin{aligned}
& \text { while }|\Omega|>N_{\text {rest }} \\
& \qquad \begin{array}{l}
k=\operatorname{argmin}_{k \in \Phi}\left(R_{k} / \lambda_{k}\right) \\
n=\operatorname{argmin}_{n \in \Omega} G_{k, n}
\end{array} \\
& \text { if } N_{k}>0 \\
& \quad c_{k, n}=1, N_{k}=N_{k}-1, \Omega=\Omega-\{n\} \\
& \quad R_{k}=R_{k}+(B / N) \log _{2}\left(1+p G_{k, n}\right) \\
& \text { else } \\
& \quad \Phi=\Phi-\{k\} \\
& \text { end }
\end{aligned}
$$

end.

Step 5: if $|\Omega|=N_{\text {rest }}$, the remaining $N_{\text {rest }}$ subcarriers are allocated to users by Hungarian algorithm, described as the following steps:

Step 5.1: construct a matrix A with size of $K \times N_{\text {rest }}$ by the subcarrier gains of $K$ users in the remaining $N_{\text {rest }}$ subcarriers.

Step 5.2: extend the matrix A with size of $K \times N_{\text {rest }}$ to a matrix $\mathbf{B}$ with size of $K \times K$ by using a zero-value virtual matrix $\mathbf{C}$ with size of $K \times\left(K-N_{\text {rest }}\right)$. That is, $\mathbf{B}=[\mathbf{A}, \mathbf{C}]$. 
Step 5.3: transform the matrix $\mathbf{B}$ into the matrix $\mathbf{M}$ by using formula $\mathbf{M}=\mathbf{B}_{\max } \times$ ones $(K, K)-\mathbf{B}$, where $\mathbf{B}_{\max }$ is the maximum value of the matrix $\mathbf{B}$ and ones $(K, K)$ is the one-value matrix with size of $K \times K$.

Step 5.4: make the matrix $\mathbf{M}$ as the input of Hungarian algorithm and output the allocation result.

Step 6: calculate and record the sum data rate $T$ and the fairness $F$. If Hungarian algorithm does not work in the searching mode, set $T_{\mathrm{op}}=T, F_{\mathrm{op}}=F, N_{\mathrm{op}}=N^{*}$ and go to Step 8; otherwise, go to Step 7.

Step 7: this step is specialized for making Hungarian algorithm work in the searching mode.

Step 7.1: record the current optimal results of $T_{\text {op }}$ and $F_{\text {op }}$ obtained in the searching process, shown below:

$$
\begin{aligned}
& \text { if } N_{\text {rest }}==N^{*} \\
& \quad T_{\text {op }}=T, F_{\text {op }}=F, N_{\text {op }}=N_{\text {rest }}, F^{*}=F \\
& \text { else } \\
& \quad \text { if }\left(T_{\text {op }} \leq T\right) \text { and }\left(F^{*} \leq F\right) \\
& \quad T_{\text {op }}=T, F_{\text {op }}=F, N_{\text {op }}=N_{\text {rest }} \\
& \text { end }
\end{aligned}
$$

end.

Step 7.2: in order for searching, set $k$ as $K-\left(N_{\text {rest }}-N^{*}-1\right)$ and modify $N_{\text {rest }}$ and $N_{k}$ until $N_{\text {rest }}=K$, shown below:

$$
\text { if } \begin{aligned}
& N_{\text {rest }}<K \\
& N_{\text {rest }}=N_{\text {rest }}+1, \Omega=\Omega^{*} \\
& k=K-\left(N_{\text {rest }}-N^{*}-1\right), N_{k}=N_{k}-1
\end{aligned}
$$

Go to Step 4 else

\section{Go to Step 8}

end.

Step 8: output the optimal results of $T_{\text {op }}, F_{\text {op }}$, and $N_{\text {op}}$.

In our proposed subcarrier allocation, the first $\left(N-N_{\text {rest }}\right)$ subcarriers are allocated through Steps 1-4 which are similar to those of the greedy method in [19] and can balance increasing the sum data rate and achieving the proportional fairness.

Different from the greedy method in [19], the remaining $N_{\text {rest }}$ subcarriers are allocated in Step 5 by the globally optimal Hungarian algorithm. Because it is from the viewpoint of global optimization that Hungarian algorithm allocates the remaining subcarriers, the proposed method is more likely to obtain higher sum data rate.

In addition, Hungarian algorithm can work in the searching mode defined in Step 7. In the searching mode, the number of the remaining subcarriers $N_{\text {rest }}$ varies from $N^{*}+1$ to $K$, and Hungarian algorithm is used to allocate the remaining $N_{\text {rest }}$ subcarriers to search the optimal results of $T_{\text {op }}$ and $F_{\text {op }}$. Note that as $N_{\text {rest }}$ increases from $N^{*}+1$ to $K, N_{k}$ with $k=K-\left(N_{\text {rest }}-N^{*}-1\right)$ is reduced by one. Through such a search for the optimal results of $T_{\text {op }}$ and $F_{\text {op }}$, both the sum data rate and the fairness can be further enhanced.
For convenience, the proposed subcarrier allocation scheme consisting of Steps 1-6 is denoted as GH, while that consisting of Steps 1-8 is denoted as GHS. Compared to the proposed $\mathrm{GH}$, the proposed GHS enables Hungarian algorithm to work in the searching mode for further improvements on the sum data rate and the proportional fairness.

4.2. Proposed ABC-Based Power Allocation for Ensuring Required Level of Proportional Fairness. In this paper, the $\mathrm{ABC}$ is used to solve power allocation for ensuring fairness because of its fewer adjustable parameters and strong global optimization ability $[15,24]$. Note that the proposed ABC based power allocation for ensuring fairness in this paper is used only if the fairness obtained by subcarrier allocation cannot achieve the required level of proportional fairness.

To reduce computational load, we set $p_{k, n}=\left(p_{k} /\left|N_{k}\right|\right)$ $(n=1,2, \ldots, N)$, where $N_{k}$ is the set of subcarriers allocated to user $k$, and define the power vector, i.e., $\mathbf{p}=\left\{p_{1}, p_{2}, \ldots, p_{K}\right\}$ as food source variable. Hence, power allocation can be transformed into the following equations:

$$
\max _{p_{k}} \sum_{k=1}^{K} \sum_{n \in N_{k}} \frac{B}{N} \log _{2}\left(1+\frac{p_{k}}{\left|N_{k}\right|} G_{k, n}\right),
$$

subject to the following constraints:

$$
\begin{aligned}
& g_{1}(\mathbf{p})=P_{T}-\sum_{k=1}^{K} p_{k} \geq 0, \quad p_{k} \geq 0, \forall k \\
& g_{2}(\mathbf{p})=F-\varepsilon \geq 0 .
\end{aligned}
$$

4.2.1. Proposed Objective Function and Fitness Function. Different from the previous work in $[17,18,26,27]$, we propose the following objective function (15) for the ABCbased power allocation:

$$
f(\mathbf{p})= \begin{cases}f_{0}+\left|g_{1}(\mathbf{p})\right|, & \text { if } g_{1}(\mathbf{p})<0, \\ f_{1}-F, & \text { if } g_{1}(\mathbf{p}) \geq 0 \text { and } g_{2}(\mathbf{p})<0, \\ -T(\mathbf{p}), & \text { if } g_{1}(\mathbf{p}) \geq 0 \text { and } g_{2}(\mathbf{p}) \geq 0,\end{cases}
$$

where $f_{0}$ and $f_{1}$ are positive real numbers satisfying $f_{0} \geq f_{1}>F$, and $T(\mathbf{p})$ is shown below:

$$
T(\mathbf{p})=\sum_{k=1}^{K} \sum_{n \in N_{k}} \frac{B}{N} \log _{2}\left(1+\frac{p_{k}}{\left|N_{k}\right|} G_{k, n}\right) .
$$

Based on the principle that a preferred solution possesses a larger fitness, the fitness function can be formulated as

$$
\operatorname{Fit}(\mathbf{p})= \begin{cases}\frac{1}{(1+f(\mathbf{p}))}, & \text { if } f(\mathbf{p}) \geq 0, \\ 1+|f(\mathbf{p})|, & \text { if } f(\mathbf{p})<0\end{cases}
$$


The proposed objective function (15) along with the fitness function (17) implies the following rules, listed as follows:

Rule 1: as for $g_{1}(\mathbf{p})<0, f(\mathbf{p})=f_{0}+\left|g_{1}(\mathbf{p})\right|$ and $\operatorname{Fit}(\mathbf{p})=1 /$ $(1+f(\mathbf{p}))$, that is, an unfeasible solution $\mathbf{p}_{1}$ with smaller violation of $g_{1}\left(\mathbf{p}_{1}\right)$ is superior to $\mathbf{p}_{2}$ with larger violation of $g_{1}\left(\mathbf{p}_{2}\right)$ because $\operatorname{Fit}\left(\mathbf{p}_{1}\right)>\operatorname{Fit}\left(\mathbf{p}_{2}\right)$ comes into existence for $g_{1}\left(\mathbf{p}_{2}\right)<g_{1}\left(\mathbf{p}_{1}\right)<0$.

Rule 2: as for $g_{1}(\mathbf{p}) \geq 0$ and $g_{2}(\mathbf{p})<0, f(\mathbf{p})=f_{1}-F$ and $\operatorname{Fit}(\mathbf{p})=1 /(1+f(\mathbf{p}))$. This means an unfeasible solution $\mathbf{p}_{1}$ with smaller violation of only $g_{2}\left(\mathbf{p}_{1}\right)$ is superior to $\mathbf{p}_{2}$ with larger violation of only $g_{2}\left(\mathbf{p}_{2}\right)$ because $\operatorname{Fit}\left(\mathbf{p}_{1}\right)$ $>\operatorname{Fit}\left(\mathbf{p}_{2}\right)$ comes into existence for $g_{2}\left(\mathbf{p}_{2}\right)<g_{2}\left(\mathbf{p}_{1}\right)<0$, $g_{1}\left(\mathbf{p}_{1}\right) \geq 0$, and $g_{1}\left(\mathbf{p}_{2}\right) \geq 0$.

Rule 3: an unfeasible solution $\mathbf{p}_{1}$ only violating the constraint of $g_{2}\left(\mathbf{p}_{1}\right)$ is superior to $\mathbf{p}_{2}$ violating the constraint of $g_{1}\left(\mathbf{p}_{2}\right)$ because $\operatorname{Fit}\left(\mathbf{p}_{1}\right)>\operatorname{Fit}\left(\mathbf{p}_{2}\right)$ comes into existence for $g_{1}\left(\mathbf{p}_{2}\right)<0, g_{2}\left(\mathbf{p}_{1}\right)<0, g_{1}\left(\mathbf{p}_{1}\right) \geq 0, f_{0} \geq f_{1}>F, f\left(\mathbf{p}_{1}\right)=f_{1}-F$, and $f\left(\mathbf{p}_{2}\right)=f_{0}+\left|g_{1}\left(\mathbf{p}_{2}\right)\right|$ and $\operatorname{Fit}(\mathbf{p})=1 /(1+f(\mathbf{p}))$.

Rule 4: as for $g_{1}(\mathbf{p}) \geq 0$ and $g_{2}(\mathbf{p}) \geq 0, f(\mathbf{p})=-T(\mathbf{p})$ and $\operatorname{Fit}(\mathbf{p})=1+|f(\mathbf{p})|$. This indicates that a feasible solution is superior to an unfeasible solution and that a feasible solution with a larger $T(\mathbf{p})$ is superior to the one with a smaller $T(\mathbf{p})$.

The above rules suggest that the proposed objective function (15) along with the fitness function (17) can guide food sources to converge from infeasible regions to feasible regions and finally to a suboptimal solution.

4.2.2. Proposed ABC-Based Power Allocation for Ensuring Required Fairness. The detailed steps of the proposed ABC for power allocation can be described as follows:

Step 1: treat power vector $\mathbf{p}$ as food source and initialize the following parameters: lower and upper bound of food source (lb and $\mathrm{ub}$ ), dimensionality of food source $(D)$, size of population $(S)$, positive constants $\left(f_{0}, f_{1}\right.$ and $\delta)$, scout production period (SPP) [15], predetermined number of cycles (Limit) to abandon food sources, the nonimprovement number $\left(\chi_{i}\right)$ of the continuous exploit on the food source $i$, the maximum number of cycles (MaxCycle), the number of the current cycle (Cycle), and the required level of proportional fairness $(\varepsilon)$.

Step 2: generate $S$ random food sources. Calculate the fitness $\operatorname{Fit}\left(\mathbf{p}_{i}\right)$ and fairness $F\left(\mathbf{p}_{i}\right)$ according to (15)-(17) and (4). Find the best food source, i.e., the food source with the maximum fitness.

Step 3: phase of employed bees. The ith employed bee uses (18) [15] to perform neighbor search for the food source $\boldsymbol{p}_{i}$ and obtain the new food source $\mathbf{v}_{i}(i \in\{1,2$, $\ldots, S\})$. If $\operatorname{Fit}\left(\mathbf{v}_{i}\right)>\operatorname{Fit}\left(\mathbf{p}_{i}\right)$, then $\mathbf{p}_{i}=\mathbf{v}_{i}, \operatorname{Fit}\left(\mathbf{p}_{i}\right)=\operatorname{Fit}\left(\mathbf{v}_{i}\right)$, $F\left(\mathbf{p}_{i}\right)=F\left(\mathbf{v}_{i}\right)$, and $\chi_{i}=0$; otherwise, $\mathbf{p}_{i}$ remains unchanged and $\chi_{i}=\chi_{i}+1$ :

$$
v_{i j}= \begin{cases}p_{i j}+\theta_{i j}\left(p_{i j}-p_{s j}\right), & \text { if } r_{j}<\delta, \\ p_{i j}, & \text { otherwise, }\end{cases}
$$

where $s$ is randomly selected from $\{1,2, \ldots, S\}, S$ is the size of population, $r_{j} \in[0,1]$ and $\theta_{i j} \in[-1,1]$ are uniformly distributed random real numbers, and $\delta \in[0,1]$ is the predetermined real number. Noted that it is with a probability of $\delta$ that the neighbor search is performed for each dimension $j(j \in\{1,2, \ldots, K\})$ of the food source $i(i \in\{1,2, \ldots, S\})$.

Step 4: evaluate the selection probability of each food source by using

$$
\operatorname{prob}_{i}= \begin{cases}\frac{1}{2}\left(1+\frac{\operatorname{Fit}\left(\mathbf{p}_{i}\right)}{\sum_{j=1}^{S} \operatorname{Fit}\left(\mathbf{p}_{j}\right)}\right), & \text { if Fit }\left(\mathbf{p}_{i}\right)>1, \\ \frac{1}{2}\left(1-\frac{\operatorname{Fit}\left(\mathbf{p}_{i}\right)}{\sum_{j=1}^{S} \operatorname{Fit}\left(\mathbf{p}_{j}\right)}\right), & \text { otherwise. }\end{cases}
$$

Step 5: phase of onlooker bees. Onlooker bees select food sources in the way of roulette wheel selection based on the selection probability (19). Assume that there exist $n_{i}$ onlooker bees who have selected the food source $\mathbf{p}_{i}(i \in\{1,2, \ldots, S\})$. Then, each of the $n_{i}$ onlooker bees uses (18) to perform neighbor search for the selected food source $\mathbf{p}_{i}$ and obtain the new food source $\mathbf{v}_{i}$. If $\operatorname{Fit}\left(\mathbf{v}_{i}\right)>\operatorname{Fit}\left(\mathbf{p}_{i}\right)$, then $\mathbf{p}_{i}=\mathbf{v}_{i}, \operatorname{Fit}\left(\mathbf{p}_{i}\right)=\operatorname{Fit}\left(\mathbf{v}_{i}\right), F\left(\mathbf{p}_{i}\right)$ $=F\left(\mathbf{v}_{i}\right)$, and $\chi_{i}=0$; otherwise, $\mathbf{p}_{i}$ remains unchanged and $\chi_{i}=\chi_{i}+1$.

Step 6: phase of scout bees. If the number of the current cycle Cycle satisfies mod (Cycle, SPP) $=0$ and there exist food sources with $\chi_{i}>$ Limit, then replace one of the food sources with a new one randomly generated by the scout bee.

Step 7: update the best food source.

Step 8: stop and output the best food source if Cycle $>$ MaxCycle. Otherwise, Cycle $=$ Cycle +1 and go to Step 3.

\section{Simulations and Discussions}

In the simulation, we consider such an OFDMA system wherein the number of subcarriers is 64 , the total bandwidth is $1 \mathrm{MHz}$, the total transmit power of the base station is $1 \mathrm{~W}$, and the power spectrum density of the noise is $-80 \mathrm{dBW} / \mathrm{Hz}$. In order to fully evaluate the performance of different algorithms, the number of users $K$ varies from 6 to 28 , and 200 sets of independent subcarrier gains $h_{k, n}$ for each $K$ are generated randomly with Rayleigh distribution. In addition, comparisons are made based on mean values of results.

\subsection{Simulations of Subcarrier Allocation}

5.1.1. Comparisons among Proposed GH, Proposed GHS, and Greedy Methods. In simulation comparisons, the greedy algorithm [19] with the equal power allocation is denoted as Greedy-EP, while that with the linear power allocation named as LINEAR in [19] is denoted as Greedy-LP in this paper. 
To be more convincing, the data rate proportionalities, i.e., $\lambda_{1}: \lambda_{2}: \cdots: \lambda_{K}=1: 1: \cdots: 1, \lambda_{1}: \lambda_{2}: \cdots: \lambda_{K}=8: 1: \cdots: 1$, and $\lambda_{1}: \lambda_{2}: \cdots: \lambda_{K}=16: 1: \cdots: 1$, are used in simulations.

For comparisons and observations, we plot difference between sum data rates of the proposed GH/GHS and Greedy-EP, the optimal number of the remaining subcarriers obtained by the proposed GH/GHS, and difference between fairness of the proposed GH/GHS and Greedy-EP in Figures $2-4$ as $K$ varies from 6 to 28. In Figures $2-4, \Delta T$ is the difference between sum data rates; $T$ (Proposed $\mathrm{GH}$ ), $T$ (Proposed GHS), and $T$ (Greedy-EP) are sum data rates obtained by the proposed $\mathrm{GH}$, the proposed GHS, and Greedy-EP, respectively. $N_{\text {op }}$ is the optimal number of the remaining subcarriers; $N_{\text {op }}$ (Proposed GH) and $N_{\text {op }}$ (Proposed GHS) are the optimal number of the remaining subcarriers obtained by the proposed $\mathrm{GH}$ and the proposed GHS, respectively. $F$ is the fairness; $\Delta F$ is the difference between fairness. Note that $N_{\text {op }}$ (Proposed GH) $=N^{*}$ while $N_{\text {op }}$ (Proposed GHS) $\geq N^{*}$, where $N^{*}$ defined in (11) is the number of the remaining subcarriers in both Greedy-EP and Greedy-LP.

From Figures 2-4(a) and 4(c) it can be seen obviously that difference between sum data rates of GH and Greedy-EP (i.e., $T$ (Proposed GH) - T(Greedy-EP)) is always larger than or equal to zero while difference between fairness of the proposed GH and Greedy-EP (i.e., F(Proposed GH) $F($ Greedy-EP) $)$ is always close to zero, which indicates that the proposed $\mathrm{GH}$ can obtain higher sum data rates than Greedy-EP while maintaining similar fairness to Greedy-EP for all of the three data rate proportionalities. In addition, from Figures 2-4(a) and 4(b), we can observe that the blue curve of $T$ (Proposed GH) - T(Greedy-EP) in Figures 2-4(a) has a similar change tendency to that of $N_{\text {op }}$ (Proposed GH) in Figures 2-4(b), which suggests that the sum data rate obtained by the proposed $\mathrm{GH}$ increases as $N_{\text {op }}$ (Proposed $\mathrm{GH}$ ) increases. The main reason is that the globally optimal Hungarian algorithm is superior to the locally optimal greedy method, and the advantage of the globally optimal Hungarian algorithm over the locally optimal greedy method is enlarged as $N_{\text {op }}$ (Proposed GH) increases.

However, the sum data rate obtained by the proposed $\mathrm{GH}$ is same with that obtained by Greedy-EP as $N^{*}$ is equal or close to zero. This is caused by the following aspects. On the one hand, $N_{\text {op }}$ (Proposed GH) of the proposed GH is always equal to $N^{*}$ of Greedy-EP. On the other hand, as $N^{*}$ is equal or close to zero, the advantage of the globally optimal Hungarian algorithm over the locally optimal greedy method fades.

Distinct with the proposed GH, the proposed GHS enables Hungarian algorithm to work in the searching mode. Positive effects of the searching mode on $N_{\text {op }}$, sum data rate, and fairness can be easily observed from Figures 2-4. As can be seen from Figures 2-4(b), in contrast with the $N_{\text {op }}$ (Proposed GH) equal or close to zero, the corresponding $N_{\text {op }}$ (Proposed GHS) obtained by the proposed GHS is more likely to be far larger than zero. Simultaneously, Figures 2-4(a) imply that the corresponding sum data rates are significantly improved by the proposed GHS, and Figures 2-4(c) indicate that the corresponding fairness is enhanced by the proposed GHS so as to be larger than those obtained by Greedy-EP. In addition, with the data rate proportionality varying from $1: 1: \cdots: 1$ to $16: 1: \cdots: 1$, maximum difference of the proposed GHS and Greedy-EP in both sum data rates and fairness becomes increasingly larger.

Comparisons between the proposed GH/GHS and Greedy-LP in sum data rates and fairness are plotted in Figures 5 and 6 as the data rate proportionality takes $1: 1$ : $\cdots: 1,8: 1: \cdots: 1$, and $16: 1: \cdots: 1$. From Figures 5 and 6 it is concluded that the proposed GH/GHS can obtain higher sum data rates while comparable rate proportional fairness for all of the three data rate proportionalities as compared with Greedy-LP. It is noted that the performance of both the proposed GH/GHS and Greedy-LP decays in sum data rates and fairness as the data rate proportionality varies from $1: 1$ : $\cdots: 1$ to $16: 1: \cdots: 1$. The main reason is that subcarriers become increasingly insufficient while allocated to users as the data rate proportionality varies from $1: 1: \cdots: 1$ to $16: 1$ : $\cdots: 1$. However, with the data rate proportionality varying from $1: 1: \cdots: 1$ to $16: 1: \cdots: 1$, the maximal gap of sum data rates between the proposed GH/GHS and Greedy-LP become increasingly larger.

From the above analyses, it can be concluded that the proposed GH/GHS outperforms both Greedy-EP and Greedy-LP and that the proposed GHS is superior to the proposed GH.

5.1.2. Comparisons between Proposed GH/GHS and Other Algorithms. In order to demonstrate the performance of our work, our proposed GH and GHS are used to compare with the other algorithms including the modified linear algorithm (modified LINEAR) [20], the artificial bee colony for only subcarrier allocation (ABC-OSA) [15], and the ant colony with constraint of the number of subcarriers (Ant-CNS) [14]. To be fair, the policy of equal power allocation is adopted by all these algorithms.

In the following simulations, only the data rate proportionality of $\lambda_{1}: \lambda_{2}: \cdots: \lambda_{K}=16: 1: \cdots: 1$ is used for better evaluating different algorithms. Besides, for comparisons and evaluations, the fairness $\varepsilon$ obtained by the proposed $\mathrm{GH}$ is used as a relaxed fairness constraint (i.e., $F \geq \varepsilon$ ) in ABC-OSA.

Sum data rates and fairness obtained by the proposed GH/GHS, modified LINEAR, ABC-OSA, and Ant-CNS are plotted in Figure 7. As can be seen from Figure 7(a), the proposed GH/GHS obtains higher sum data rates than all these algorithms. And, Figure 7(b) shows that the proposed GH/GHS can achieve acceptable proportional fairness. However, Ant-CNS suffers from a seriously decayed performance in fairness. The reason is that the number of the remaining subcarriers increases rapidly with $K$ varying from 18 to 28 as the data rate proportionality is $16: 1: \cdots: 1$.

Note that, in simulations, the fairness obtained by $\mathrm{GH}$ is used as a relaxed fairness constraint (i.e., $F \geq \varepsilon$ ) in ABC-OSA. However, as can be seen from Figure 7, sum data rates obtained by ABC-OSA are lower than all of the other algorithms, and the fairness obtained by ABC-OSA cannot exceed that obtained by $\mathrm{GH}$. The results imply that, due to insufficient 


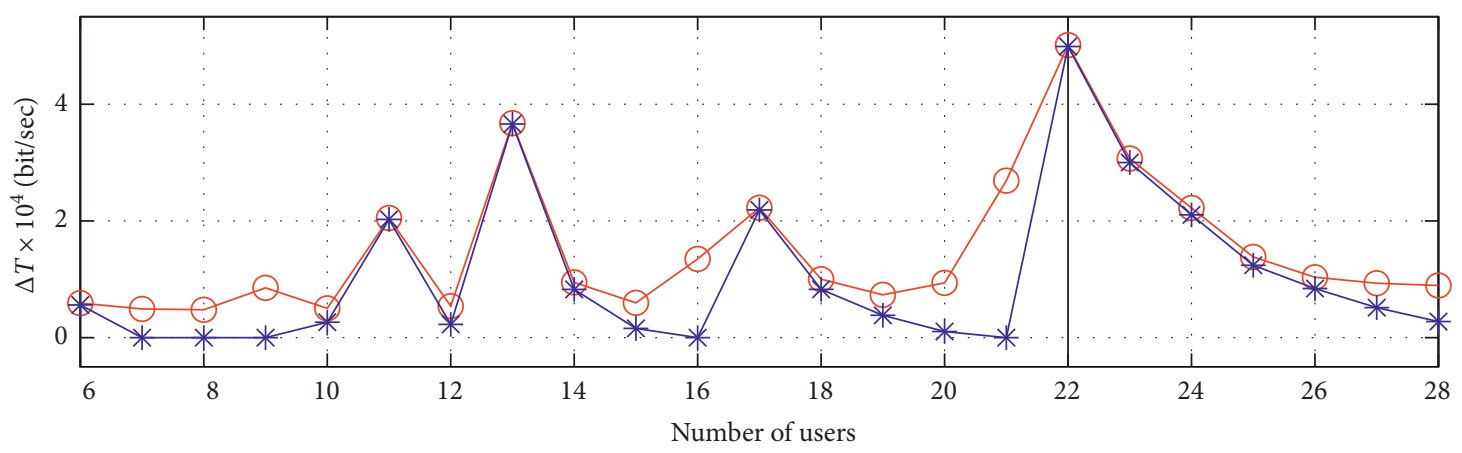

(a)

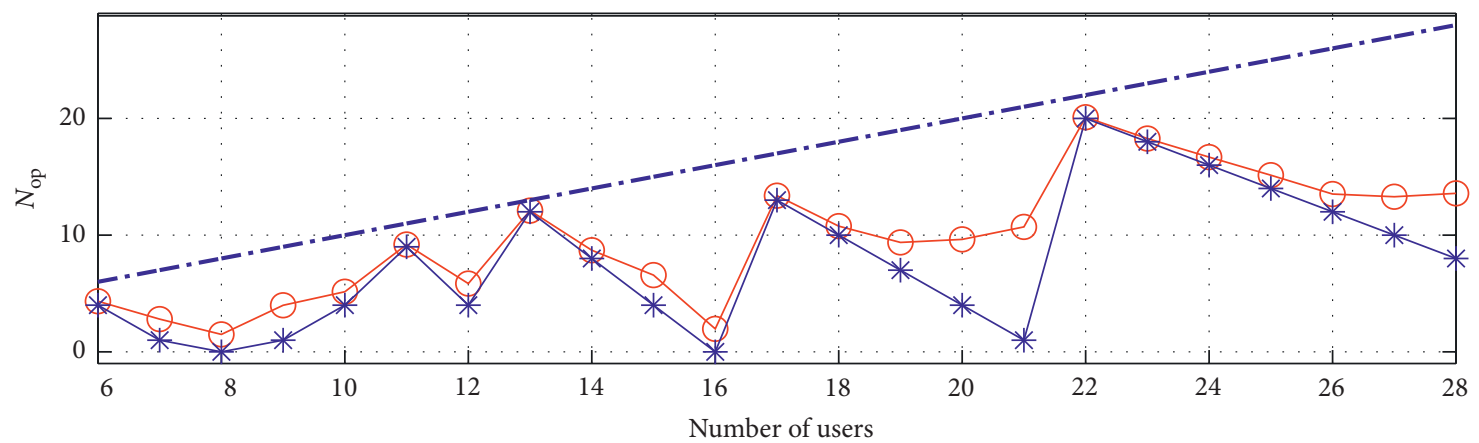

$\bigcirc N_{\text {op }}$ (Proposed GHS)
$* \quad N_{\text {op }}$ (Proposed GH)
$\cdots \cdots$

Number of users

T (Proposed GHS) - T (Greedy-EP)

* $T$ (Proposed GH) - T (Greedy-EP)

(b)

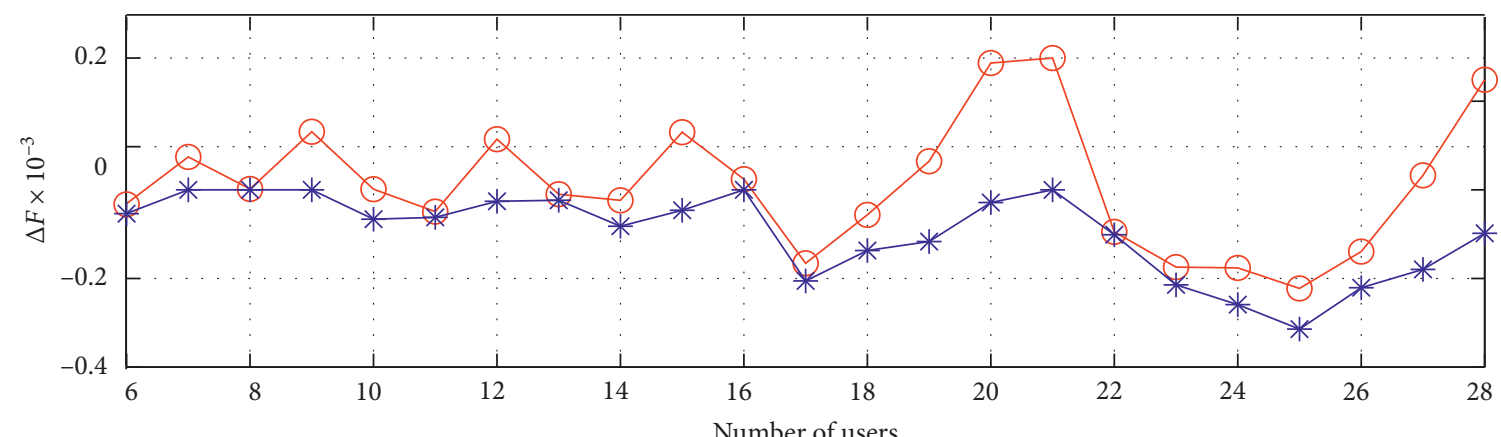

- $F$ (Proposed GHS) $-F$ (Greedy-EP)

* $F$ (Proposed GH) $-F($ Greedy-EP)

(c)

FIGURE 2: Simulation comparisons between the proposed GH/GHS and Greedy-EP as $\lambda_{1}: \lambda_{2}: \cdots: \lambda_{K}=1: 1: \cdots: 1$. (a) Difference between sum data rates of the proposed GH/GHS and Greedy-EP. (b) Optimal number of the remaining subcarriers obtained by the proposed GH/ GHS. (c) Difference between fairness of the proposed GH/GHS and Greedy-EP.

allocation of subcarriers to users, it is difficult for ABC-OSA to satisfy the relaxed fairness constraint (i.e., $F \geq \varepsilon$ ), leading to the poor performance of ABC-OSA in sum data rates.

The above analyses show that the proposed GH/GHS can acquire higher sum data rate and acceptable fairness as compared with Modified LINEAR, ABC-OSA, Ant-CNS, and Ant-NoCNS.
5.2. Simulations of Power Allocation for Ensuring Required Level of Proportional Fairness. As it is analyzed in Section 5.1, subcarriers would be likely to be insufficient in allocation to users so that the obtained fairness cannot satisfy the required fairness. Hence, the proposed ABC-based power allocation of Section 4.2 is used to ensure the required level of proportional fairness in simulations of this section as the 


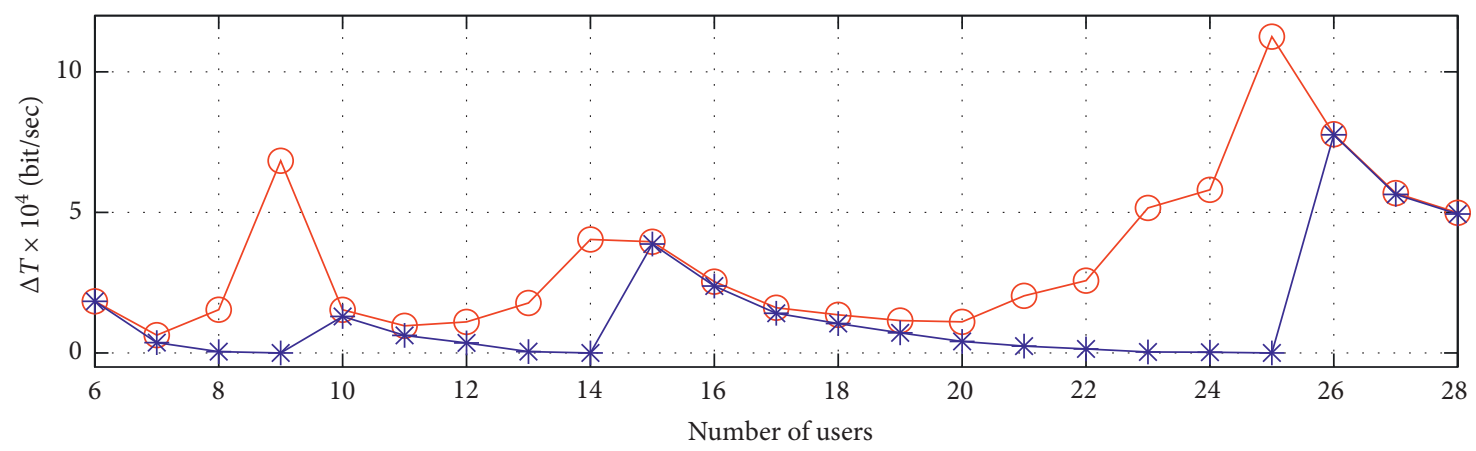

$T$ (Proposed GHS) $-T$ (Greedy-EP)

* $T$ (Proposed GH) $-T$ (Greedy-EP)

(a)

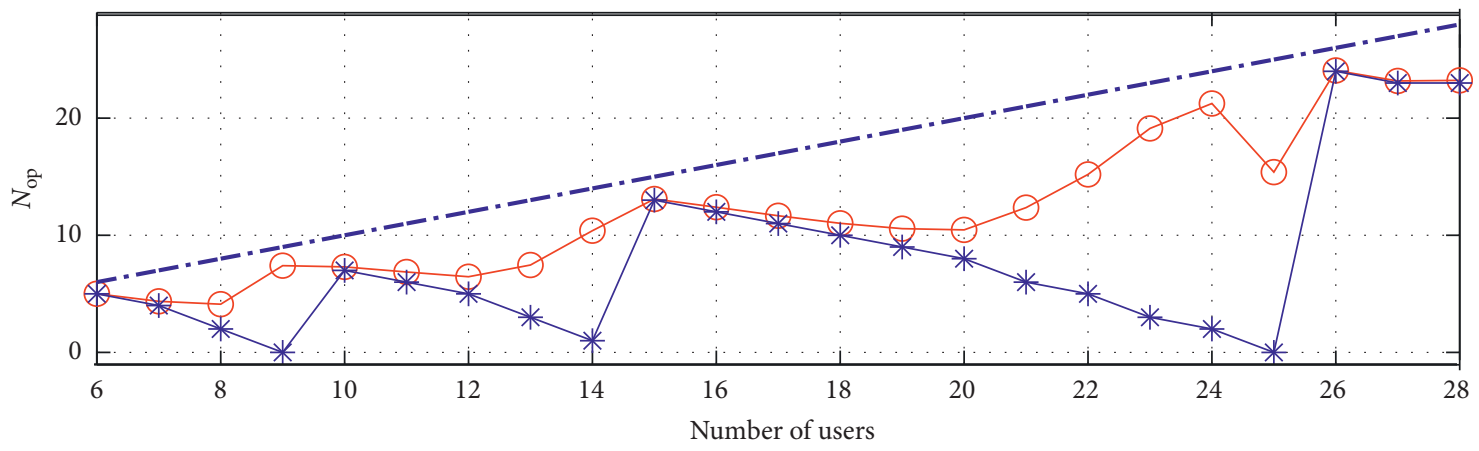

$N_{\text {op }}$ (Proposed GHS)

* $N_{\text {op }}$ (Proposed GH)

-..... $K$

(b)

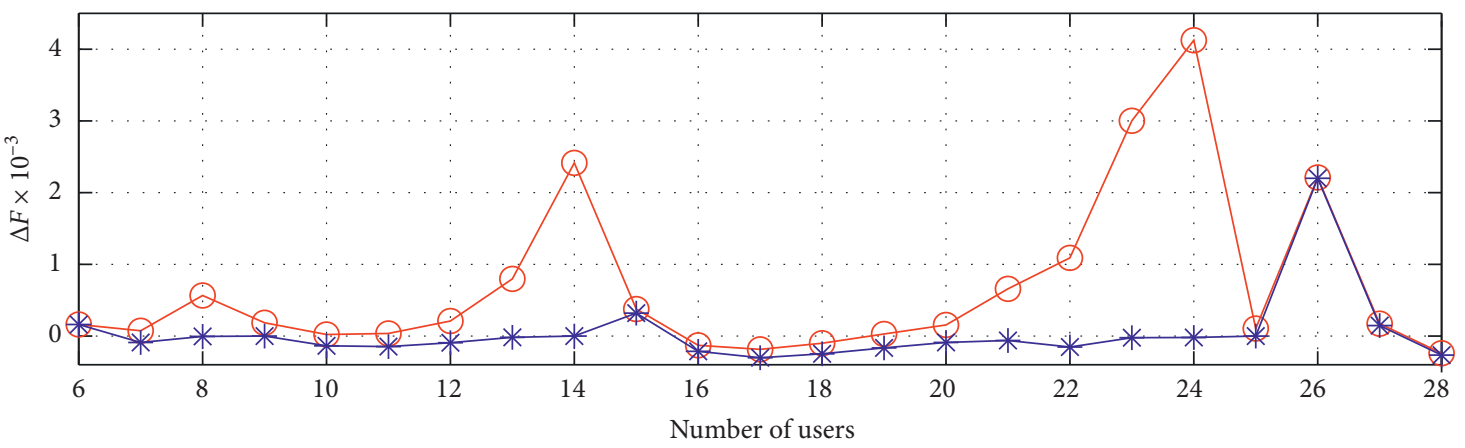

$F$ (Proposed GHS) $-F$ (Greedy-EP)

* $F$ (Proposed GH) $-F($ Greedy-EP)

(c)

FIGURE 3: Simulation comparisons between the proposed GH/GHS and Greedy-EP as $\lambda_{1}: \lambda_{2}: \cdots: \lambda_{K}=8: 1: \cdots: 1$. (a) Difference between sum data rates of the proposed GH/GHS and Greedy-EP. (b) Optimal number of the remaining subcarriers obtained by the proposed GH/ GHS. (c) Difference between fairness of the proposed GH/GHS and Greedy-EP.

data rate proportionality is $16: 1: \cdots: 1$. The parameters of the proposed $\mathrm{ABC}$ to ensure the required level of proportional fairness are set as Table 1.

5.2.1. Comparisons between the Proposed Hybrid Scheme and $A B C$ [27] with Fitness Function (9). Based on the result of subcarrier allocation obtained by GHS, the proposed
$\mathrm{ABC}$ is compared with the $\mathrm{ABC}$ [27] in power allocation for achieving the required lowest level of fairness. Note that the ABC [27] uses the fitness function (7) while the proposed ABC uses the fitness function (17) along with (15) and (16). For comparisons, the parameters of $A B C$ [27] are same with those of the proposed ABC in Table 1. 


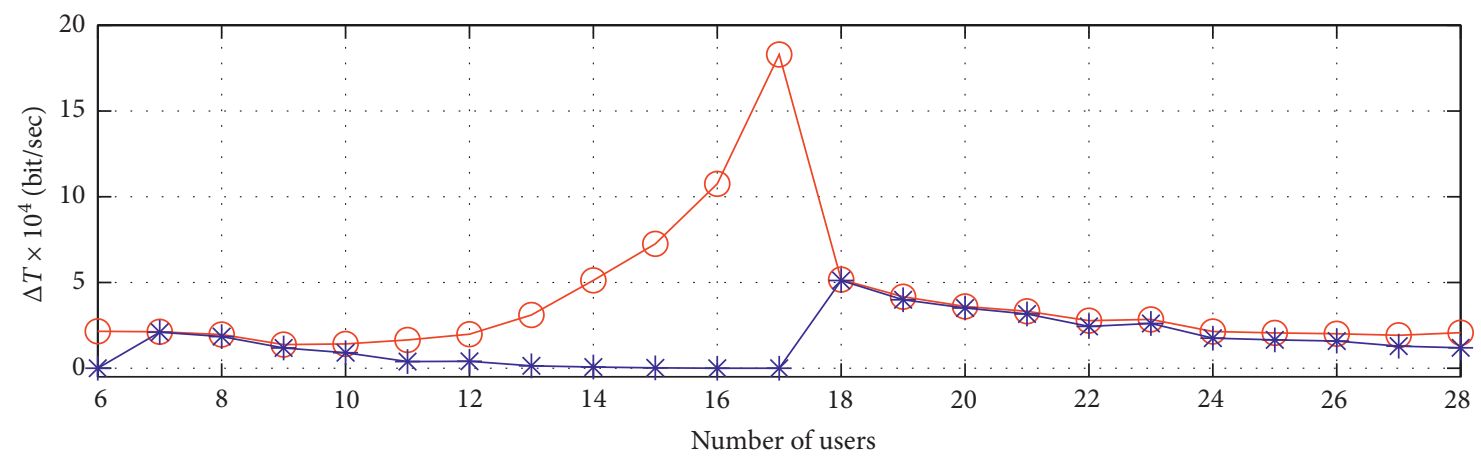

$T$ (Proposed GHS) - T (Greedy-EP)

* $T$ (Proposed GH) $-T$ (Greedy-EP)

(a)

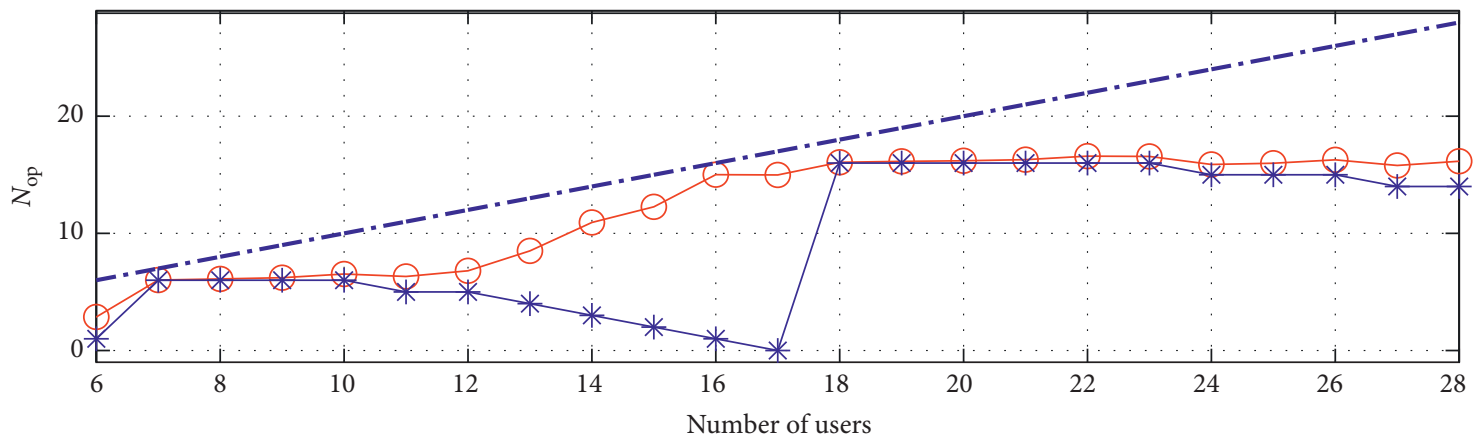

$\bigcirc N_{\text {op }}$ (Proposed GHS)

$* N_{\text {op }}$ (Proposed GH)

$\cdots \cdots$

(b)

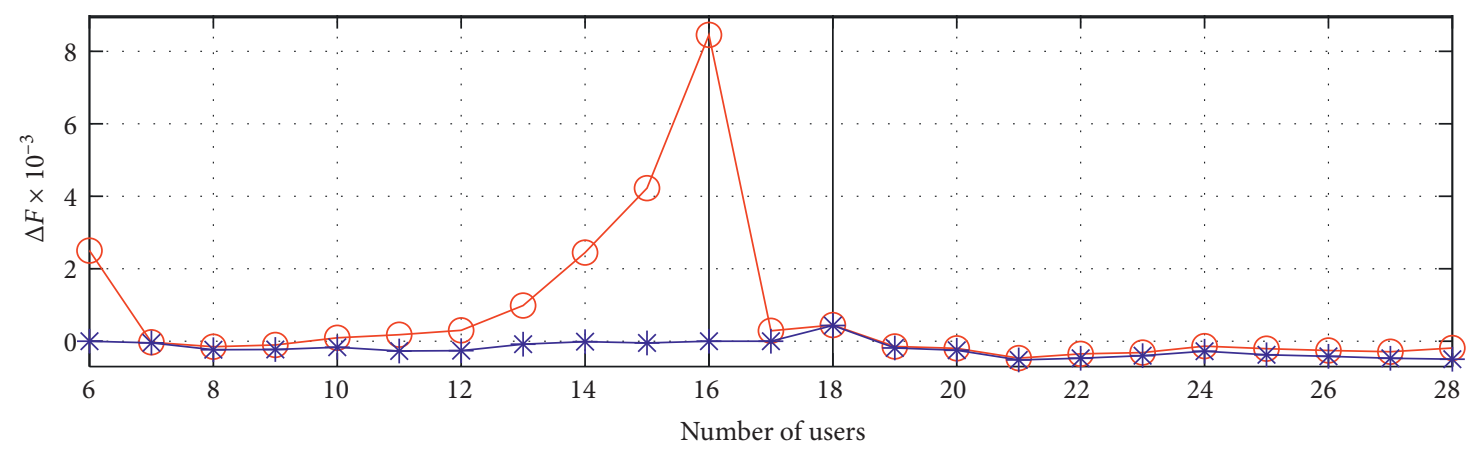

F (Proposed GHS) $-F$ (Greedy-EP)

* $F$ (Proposed GH) $-F$ (Greedy-EP)

(c)

FIGURE 4: Simulation comparisons between the proposed GH/GHS and Greedy-EP as $\lambda_{1}: \lambda_{2}: \cdots: \lambda_{K}=16: 1: \cdots: 1$. (a) Difference between sum data rates of the proposed GH/GHS and Greedy-EP. (b) Optimal number of the remaining subcarriers obtained by the proposed GH/ GHS. (c) Difference between fairness of the proposed GH/GHS and Greedy-EP.

Comparisons between the proposed $\mathrm{ABC}$ and $\mathrm{ABC}$ [27] in power allocation are made based on Figure 5. Obviously, the obtained fairness in Figure 5 is lower than the predetermined required lowest level of fairness, i.e., 0.96 , as the number of users varies from 21 to 28 . Hence, both the proposed $\mathrm{ABC}$ and $\mathrm{ABC}$ [27] are compared as the number of users varies from 21 to 28 , and the comparison results are shown in Figure 8. As is seen from Figure 8(a), the proposed $\mathrm{ABC}$ can achieve the required lowest level of fairness 0.96 by power allocation as the number of users varies from 21 to 28 , while the $\mathrm{ABC}$ [27] cannot achieve the required lowest level of fairness 0.96 as the number of users varies from 22 to 28 . And, Figure 8(b) illustrates that the proposed $A B C$ can effectively converge to the required level of proportional 


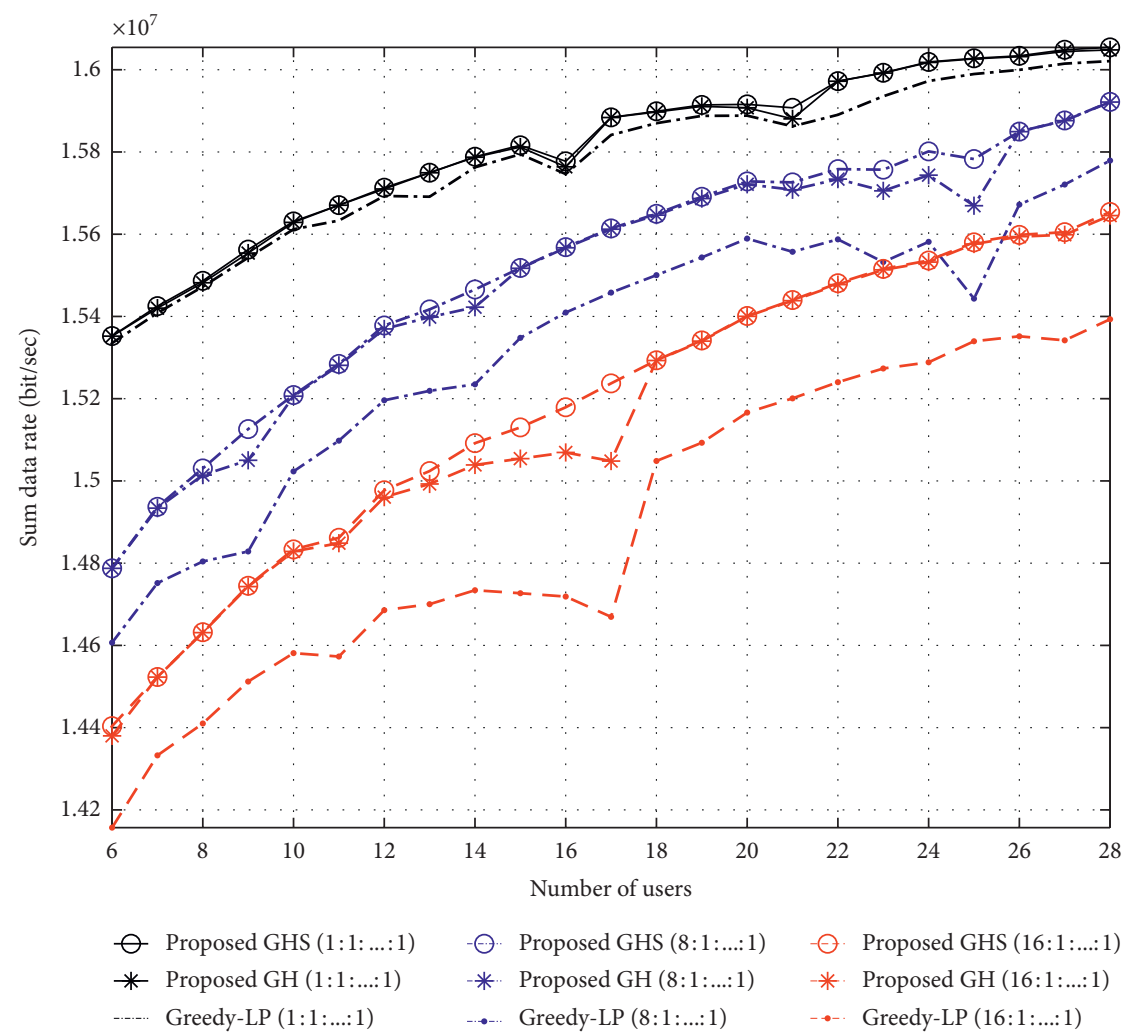

FIGURE 5: Comparisons between the proposed GH/GHS and Greedy-LP in sum data rates as the data rate proportionality takes $1: 1: \cdots: 1,8$ : $1: \cdots: 1$, and $16: 1: \cdots: 1$.

fairness within 600 iterations for a $K=28$ instance as compared with the ABC [27].

\subsubsection{Comparisons between the Proposed Hybrid Scheme and} the Fairness Threshold-Based Greedy Algorithm Combined with the PSO. In this subsection, the proposed scheme, i.e., the proposed GH/GHS combined with the proposed $\mathrm{ABC}$ is compared with the fairness threshold-based greedy algorithm combined with the PSO $[17,18]$. For convenience, the fairness threshold-based greedy algorithm is denoted as Greedy-Threshold, the fairness threshold-based greedy algorithm combined with the PSO is denoted as "GreedyThreshold+PSO," and the proposed hybrid scheme is denoted as "Proposed GH + Proposed ABC" or "Proposed GHS + Proposed ABC.”

First, fairness comparisons are made between the proposed GH/GHS and Greedy-Threshold in Figure 9 as the data rate proportionality is $16: 1: \cdots: 1$. As can be seen from Figure 9, Greedy-Threshold cannot achieve the required level of proportional fairness 0.96 as the number of users varies from 6 to 28. The main reason is that the greedy method $[17,18]$ trying to maximize the sum data rates easily destroys the proportional fairness. This means the PSObased power allocation would be used inevitably and frequently to meet the required level of proportional fairness. Different from Greedy-Threshold, it is only at the number of users of 21-28 that the proposed GH/GHS cannot achieve the required level of proportional fairness 0.96 .
Second, based on the results in Figure 9, the proposed $\mathrm{ABC}$ and the PSO $[17,18]$ are used in power allocation to ensure the required level of proportional fairness 0.96 , respectively. Simulation results depicted in Figure 10 show that all of the obtained fairness is larger than the required level of proportional fairness 0.96 , which suggests both the proposed ABC and the PSO $[17,18]$ can be able to ensure the required level of proportional fairness 0.96 in power allocation. However, sum data rates obtained by "GreedyThreshold + PSO" are obviously lower than those obtained by "Proposed $\mathrm{GH}+$ Proposed ABC" and "Proposed GHS + Proposed ABC," which is mainly caused by the premature convergence of the PSO $[17,18]$. Note that only the results at the number of users of 21-28 are obtained by using the proposed $\mathrm{ABC}$ based power allocation.

Third, further comparisons between the proposed ABC and the PSO $[17,18]$ are made based on subcarrier allocation results obtained by the proposed GHS as the data rate proportionality is $16: 1: \cdots: 1$. Both sum data rate and fairness obtained for a $K=28$ instance by PSO and the proposed $\mathrm{ABC}$ at different parameters in 100 runs are summarized in Table 2 . The involved parameters include limit value for velocities ( $\left.V_{\max }\right)$ of PSO, positive constant $\left(f_{1}\right)$ of the objective function (21) of the proposed $A B C$, and scout production period (SPP) of the proposed $\mathrm{ABC}$. In the table, $N_{F} \geq 0.96$ represents the number of runs converging to solutions with $F \geq 0.96$. Results in Table 2 indicate that both sum data rate and $N_{F} \geq 0.96$ are easily influenced by the limit value for velocities $\left(V_{\max }\right)$ so that the improper $V_{\max }$ leads to 


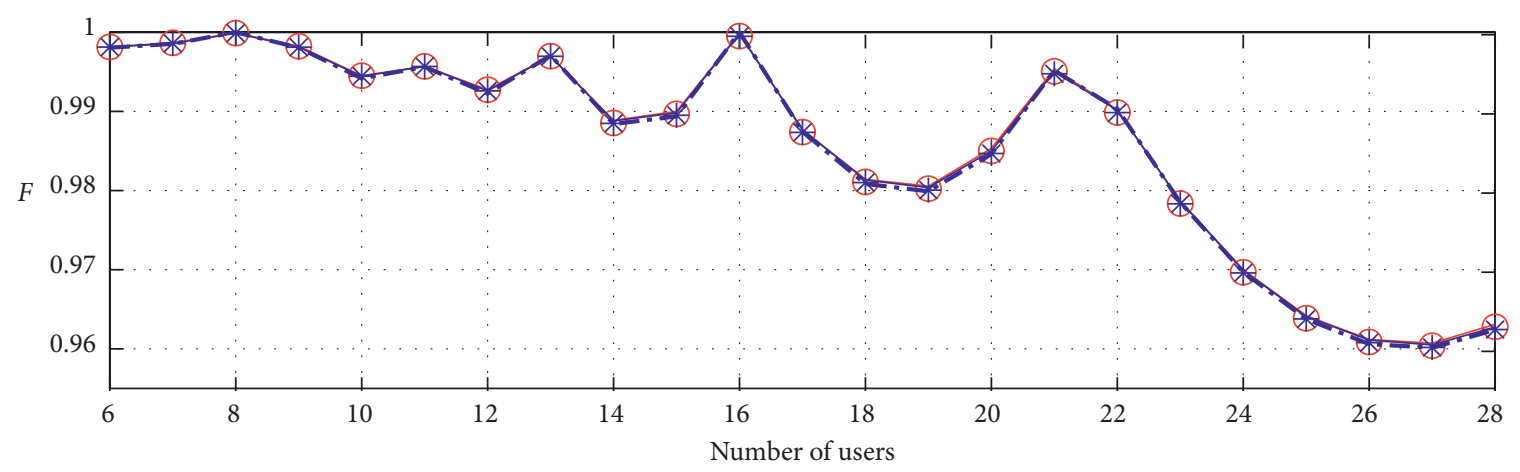

$\bigcirc$ Proposed GHS $(1: 1: \ldots: 1)$

* Proposed GH $(1: 1: \ldots: 1)$

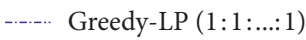

(a)

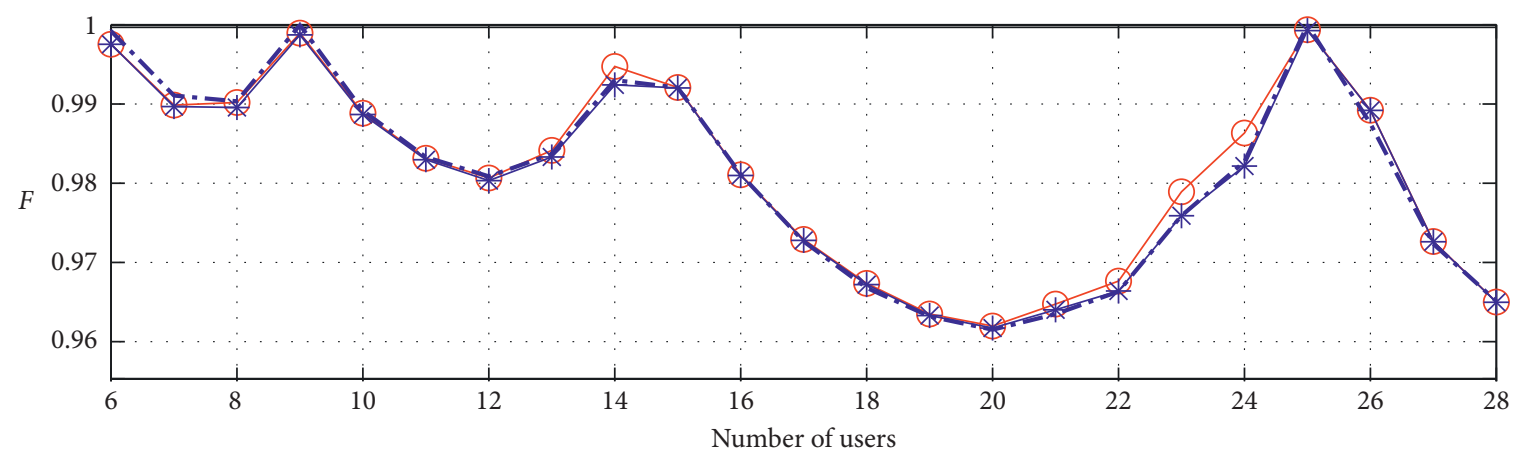

$\bigcirc$ Proposed GHS $(8: 1: \ldots: 1)$

* Proposed GH $(8: 1: \ldots: 1)$

-..... Greedy-LP $(8: 1: \ldots: 1)$

(b)

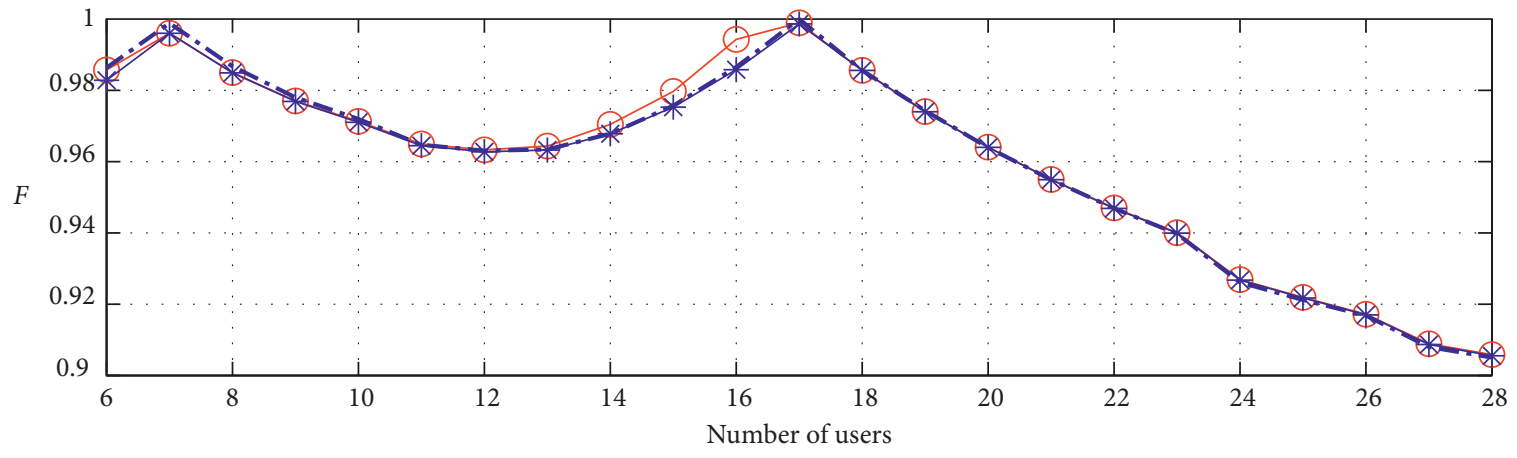

$$
\begin{aligned}
& \text { Proposed GHS }(16: 1: \ldots: 1) \\
& \text { * Proposed GH }(16: 1: \ldots .1) \\
& -\ldots \text { Greedy-LP }(16: 1: \ldots: 1)
\end{aligned}
$$

(c)

Figure 6: Comparisons between the proposed GH/GHS and Greedy-LP in fairness as the data rate proportionality takes (a) $1: 1: \cdots: 1$, (b) 8 : $1: \cdots: 1$, and (c) $16: 1: \cdots: 1$.

a decayed performance in $N_{F} \geq 0.96$. For example, $N_{F} \geq 0.96$ decreases from 100 to 17 as $V_{\max }$ increases from 0.0001 to 0.001 . Oppositely, results obtained by our proposed ABC in Table 2 suggest that $N_{F} \geq 0.96$ holds 100 as positive constants $\left(f_{1}\right)$ of the objective function (21) increases from 0.96 to 5000 and scout production period (SPP) increases from 10 to 1000. Besides, the standard deviation (std) of sum data rates obtained by our proposed $\mathrm{ABC}$ is two orders of magnitude less than that obtained by the PSO $[17,18]$. This indicates the proposed $\mathrm{ABC}$ is much steadier than the PSO $[17,18]$. 


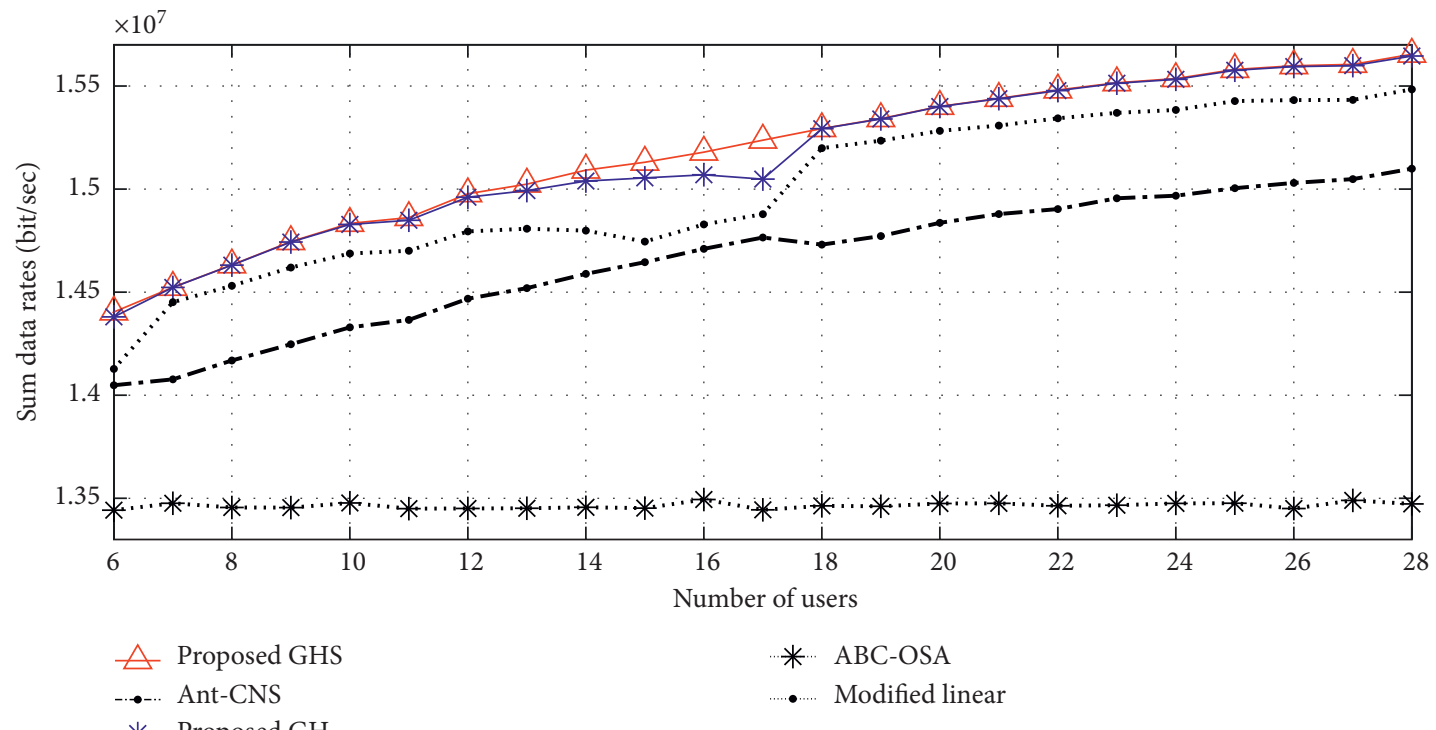

(a)

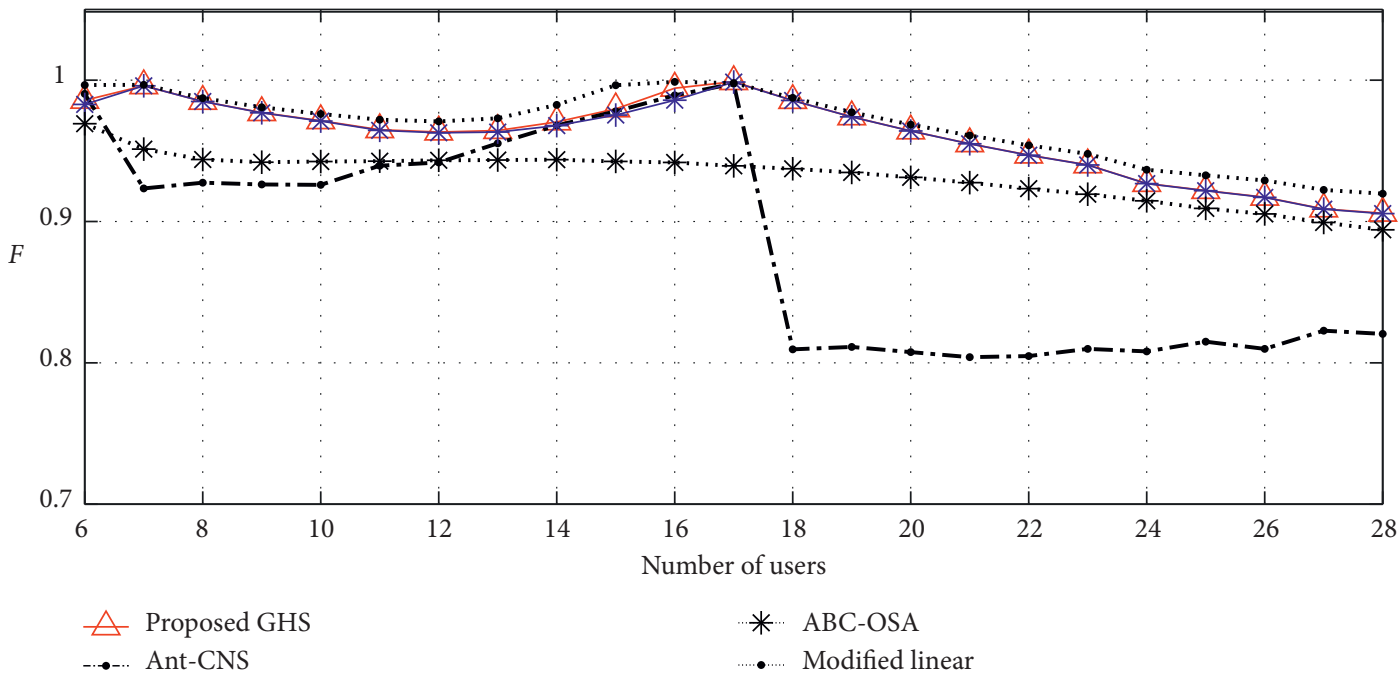

(b)

FIGURE 7: Simulation results obtained by the proposed GH/GHS and other algorithms as the data rate proportionality is $16: 1: \cdots: 1$. (a) Sum data rates obtained by the proposed GH/GHS and other algorithms. (b) Fairness obtained by the proposed GH/GHS and other algorithms.

TABle 1: Parameters of the proposed ABC.

\begin{tabular}{lc}
\hline Parameters & Values \\
\hline Lower and upper bound of food source & $\mathrm{lb}=0, \mathrm{ub}=1$ \\
Size of population & $S=80$ \\
The maximum number of cycles & MaxCycle $=2000$ \\
Positive constants & $f_{0}=5000, f_{1}=10, \delta=0.6$ \\
Scout production period & $\mathrm{SPP}=12$ \\
The predetermined number of cycles to abandon food sources & Limit $=10$ \\
\hline
\end{tabular}

Results in Table 2 show that the proposed ABC with larger $f_{1}$ or SPP could obtain higher sum data rates. All these show the superiority of our proposed ABC to the PSO $[17,18]$.
5.2.3. Comparisons between the Proposed Hybrid Scheme and the Power Allocation with Strict Proportional Fairness. In this subsection, our proposed ABC-based power allocation 


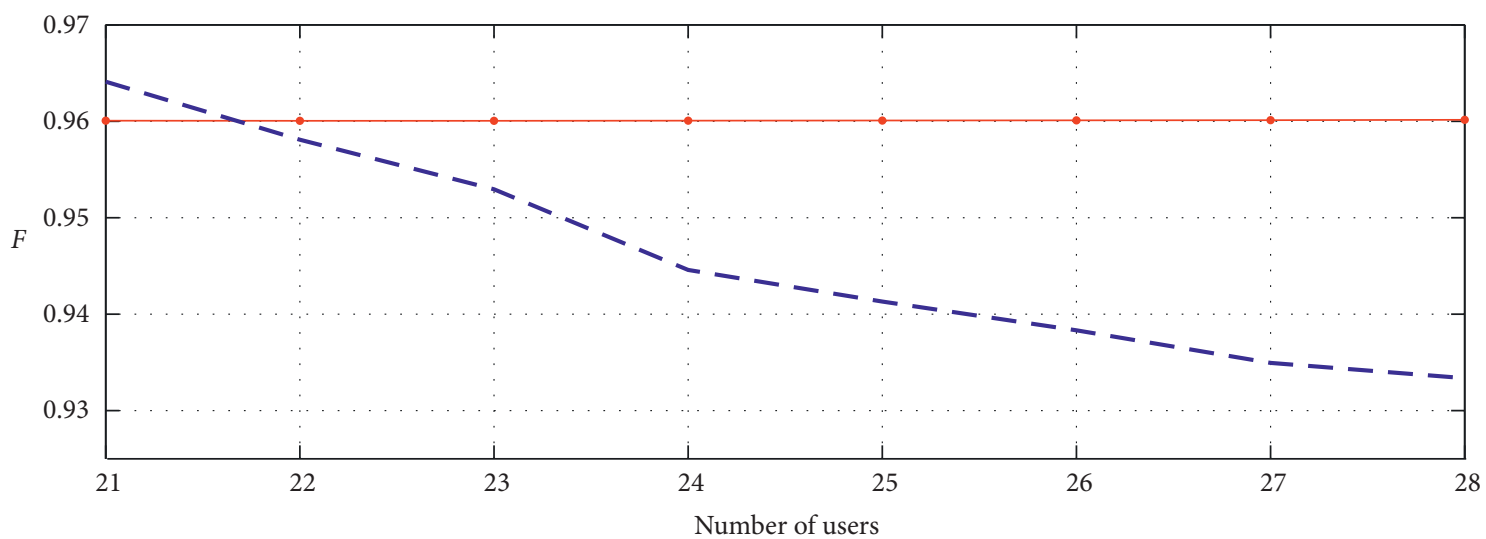

$\rightarrow$ Proposed GHS + proposed ABC

--- Proposed GHS + ABC [27]

(a)

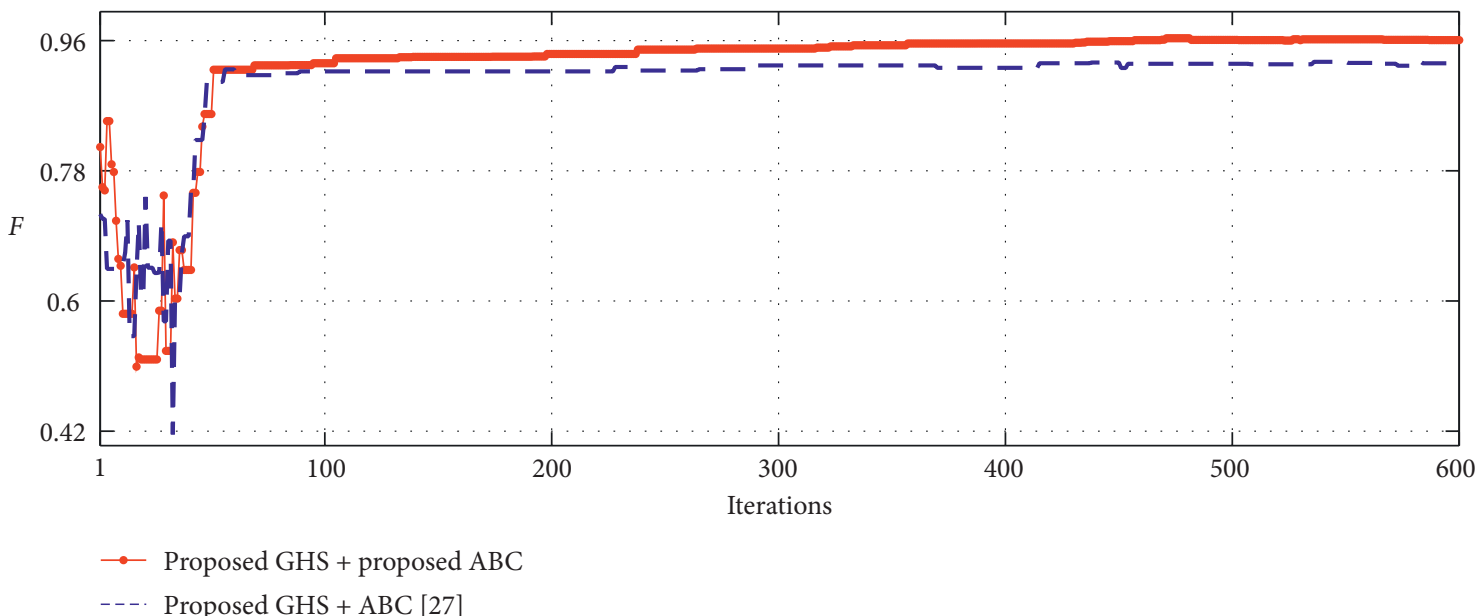

(b)

FIGURE 8: Results obtained by the proposed $A B C$ and $A B C$ [27]. (a) Fairness obtained by the proposed $A B C$ and $A B C$ [27] as the number of users varies from 21 to 28. (b) Convergent curves from the proposed $\mathrm{ABC}$ and $\mathrm{ABC}$ [27] for a $K=28$ instance.

for ensuring fairness is compared with the power allocation with strict proportional fairness [5]. For convenience, comparisons are made based on the results of subcarrier allocation obtained by the proposed GHS as the data rate proportionality is $16: 1: \cdots: 1$. Comparison results are shown in Figure 11, where "Proposed GHS with strict fairness" is the result obtained by the power allocation with strict proportional fairness [5] for subcarrier allocation obtained by the proposed GHS. It can be seen obviously that the proposed GHS with strict fairness [5] can achieve the strict fairness, i.e., $F=1$. However, the proposed GHS with strict fairness [5] obtains much lower sum data rates than our proposed ABC-based power allocation for ensuring the required level of proportional fairness 0.96 . Specially, the proposed GHS with strict fairness [5] experiences a drastic decrease in sum data rates as $K$ varies from 18 to 28 . The main reason is that subcarriers are relatively insufficient while allocated to users as $K$ varies from 18 to 28 . That is, the power allocation with strict proportional fairness [5] cannot satisfy the requirements of the new-generation wireless communications for higher sum data rates.
5.3. Complexity Analysis. Because the proposed GH consists of Steps 1-5 of Section 4.1, the complexity of the proposed $\mathrm{GH}$ can be drawn from these steps. In these steps, Steps 1 and 3 require constant time. Steps 2 and 4 are the same as those in greedy algorithm [19], requiring $\mathrm{O}\left(\mathrm{KNlog}_{2} \mathrm{~N}\right)$ and $O((N-K) * K)$ operations [19]. Step 5 has a same complexity with Hungarian algorithm of $O\left(K^{3}\right)$. If $K N \log _{2} N \geq K^{3}$, the proposed $\mathrm{GH}$ has a asymptotic complexity of $\mathrm{O}\left(\mathrm{KN} \log _{2} N\right)$; otherwise, the proposed $\mathrm{GH}$ has a asymptotic complexity of $O\left(K^{3}\right)$.

Different from the proposed $\mathrm{GH}$, the proposed GHS consists of Steps 1-8 of Section 4.1, where Steps 1, 3, 6, 7, and 8 require constant time. The searching mode of GHS enables Steps 4 and 5 to be run in cycle. Note that, as $N^{*}$ is zero, the number of cycles is $K$; otherwise, the number of cycles is smaller than $K$. That is to say, Steps 4 and 5 will run $K$ times in the worst condition of the searching mode of GHS. Therefore, the maximum asymptotic complexity of Step 4 is approximate to $O\left((N-3 *((K+1) / 2)) * K^{2}\right)$, while that of Step 5 is approximate to $O\left(K^{5}\right)$. 


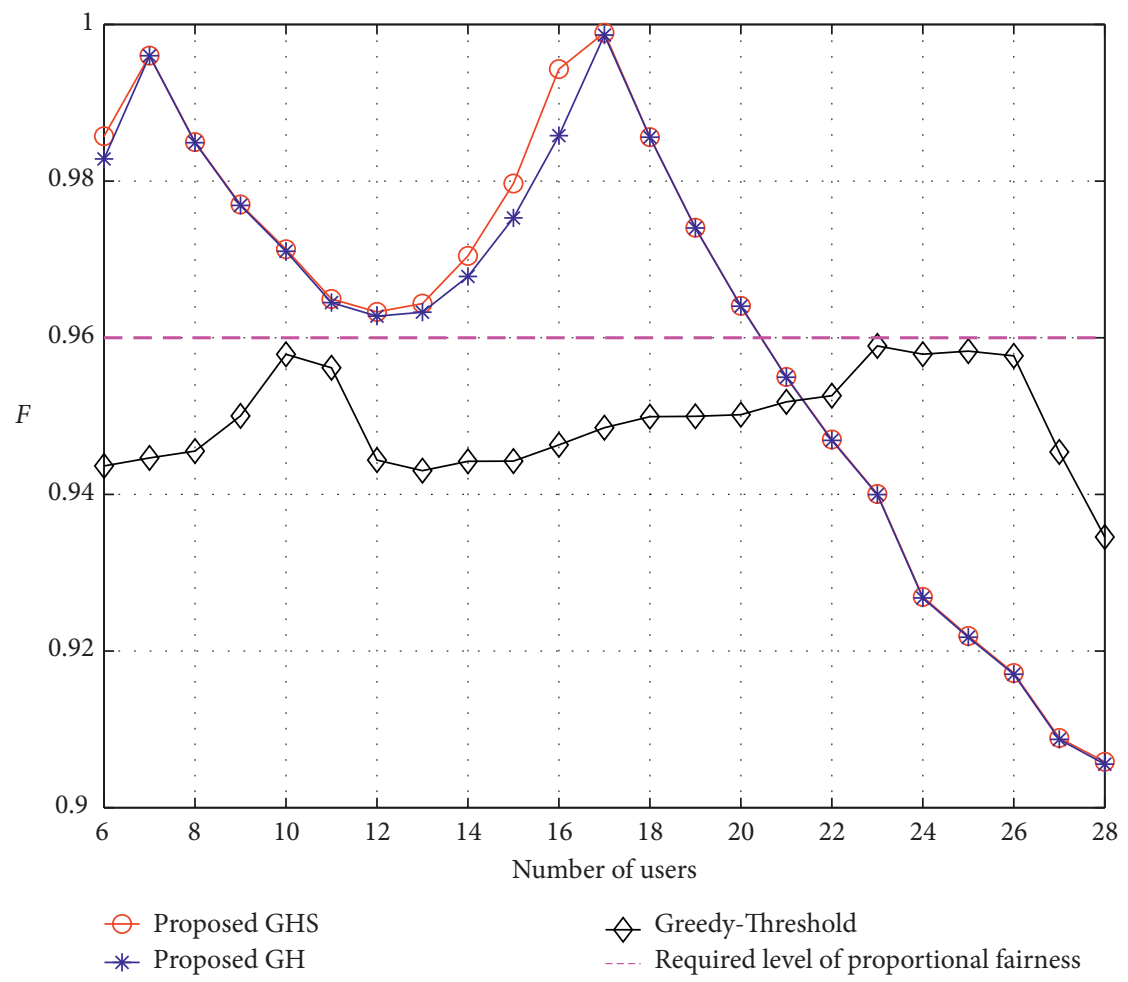

FIgURE 9: Fairness obtained by the proposed GH/GHS and Greedy-Threshold $[17,18]$ as the data rate proportionality is $16: 1: \cdots: 1$.

In the proposed ABC-based power allocation for ensuring the fairness, there are $S$ food sources and each food source has a dimensionality of $K$. Hence, in each cycle $2 * K * S$ searches in total are performed by employed bees and onlooker bees. Besides, the fitness function requires $N$ sum operations. Hence, in each cycle, $2 * N * S$ sum operations in total are performed by the fitness function. Because $N>K$, the asymptotic complexity of the proposed ABCbased power allocation is up to $O(2 * N * S *$ MaxCycle) for the maximum number of cycles of MaxCycle. Differently, both the equal power allocation and the linear power allocation have a asymptotic complexity of $O(K)$.

Complexity comparisons among different algorithms are summarized in Table 3 . It shows that the complexity of the proposed GH/GHS in subcarrier allocation may lie between that of greedy methods and that of evolutionary algorithms and that the complexity of the proposed GHS is higher than that of the proposed $\mathrm{GH}$. In addition, the proposed ABCbased power allocation for ensuring the fairness has a slightly higher complexity than the PSO-based power allocation $[17,18]$.

5.4. Discussions. From the above simulation results of Sections 5.1 and 5.2, it can be seen that the proposed GHS has advantages over the proposed $\mathrm{GH}$ in both sum data rates and fairness. However, the computational complexity of the proposed GHS is higher than that of the proposed GH. This leads the proposed GHS to take much more time in searching the better solutions for the rate adaptive resource allocation. Fortunately, it can be relieved by the following facts.
First, before GHS finishes, the solution of GH can be used as an alternative one. It can be seen from Steps 1-8 of Section 4.1 that the searching mode of GHS starts from the solution of $\mathrm{GH}$, which means that $\mathrm{GH}$ runs before GHS. Besides, it can be seen from Table 3 that $\mathrm{GH}$ is more close to greedy methods in the complexity than GHS.

Second, one can balance the tradeoff between quality of a solution and computational cost to determine whether to use GHS or not. The simulation analyses of Section 5.1 suggest that, if $N^{*}$ from (11) equals to zero or close to zero and satisfies $N^{*} \ll K$, the proposed GHS is obviously better than the proposed GH. In addition, complexity analysis of Section 5.3 indicates that the computational cost increases as the number of users of $K$, satisfying $N^{*}=0$, increases.

From the above simulation results of Sections 5.1 and 5.2 , it can be seen that the proposed GH/GHS using equal power allocation can obtain higher sum data rates while acceptable proportional fairness as subcarriers are relatively sufficient in allocation to users. As either the number of users or the imbalance of the proportional data rate constraint increases, subcarriers may be insufficient while allocated to users, which is more likely to make the obtained fairness lower than the required level of proportional fairness. The proposed ABC-based power allocation can be applied to achieve the acceptable proportional fairness. Although the proposed ABC-based power allocation has a slightly higher complexity than the PSO-based power allocation $[17,18]$, it can converge to the required level of proportional fairness but with higher sum data rates. In addition, the proposed ABC based power allocation performs more steadily than the PSO-based power allocation $[17,18]$. 


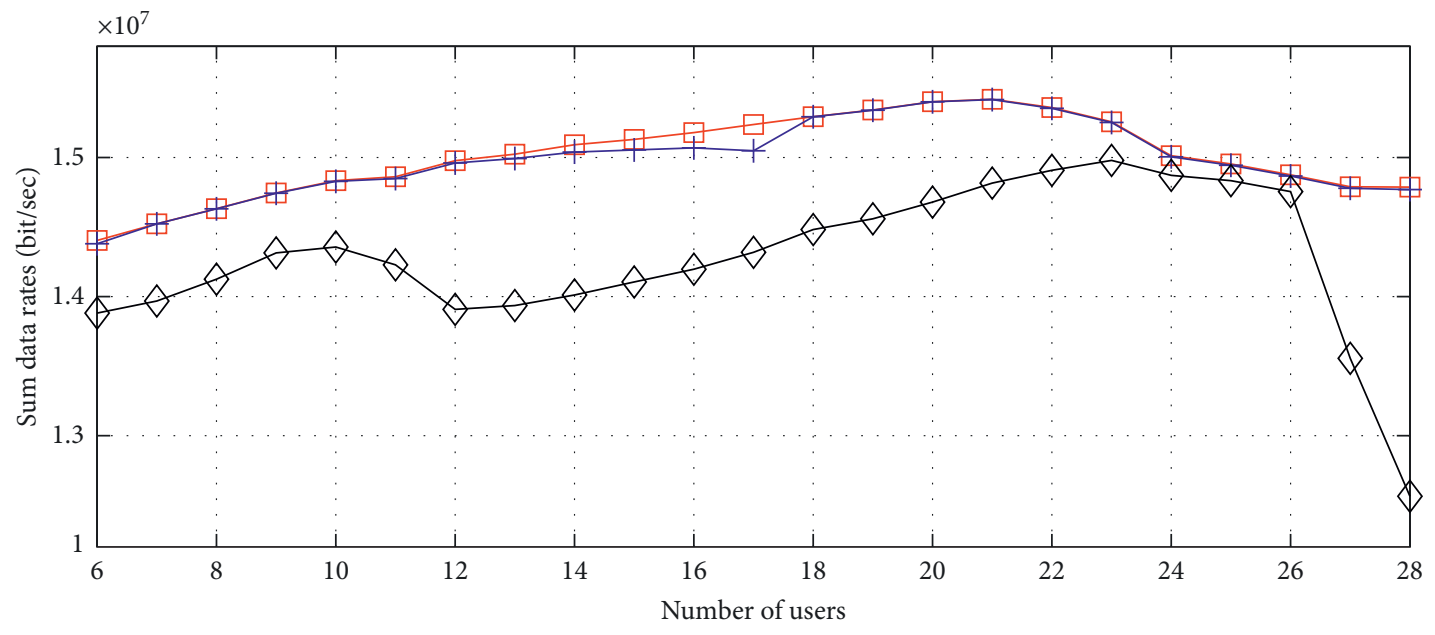

$\square$ Proposed GHS + proposed ABC

+ Proposed GH + proposed ABC

$\vartheta$ Greedy-Threshold + PSO

(a)

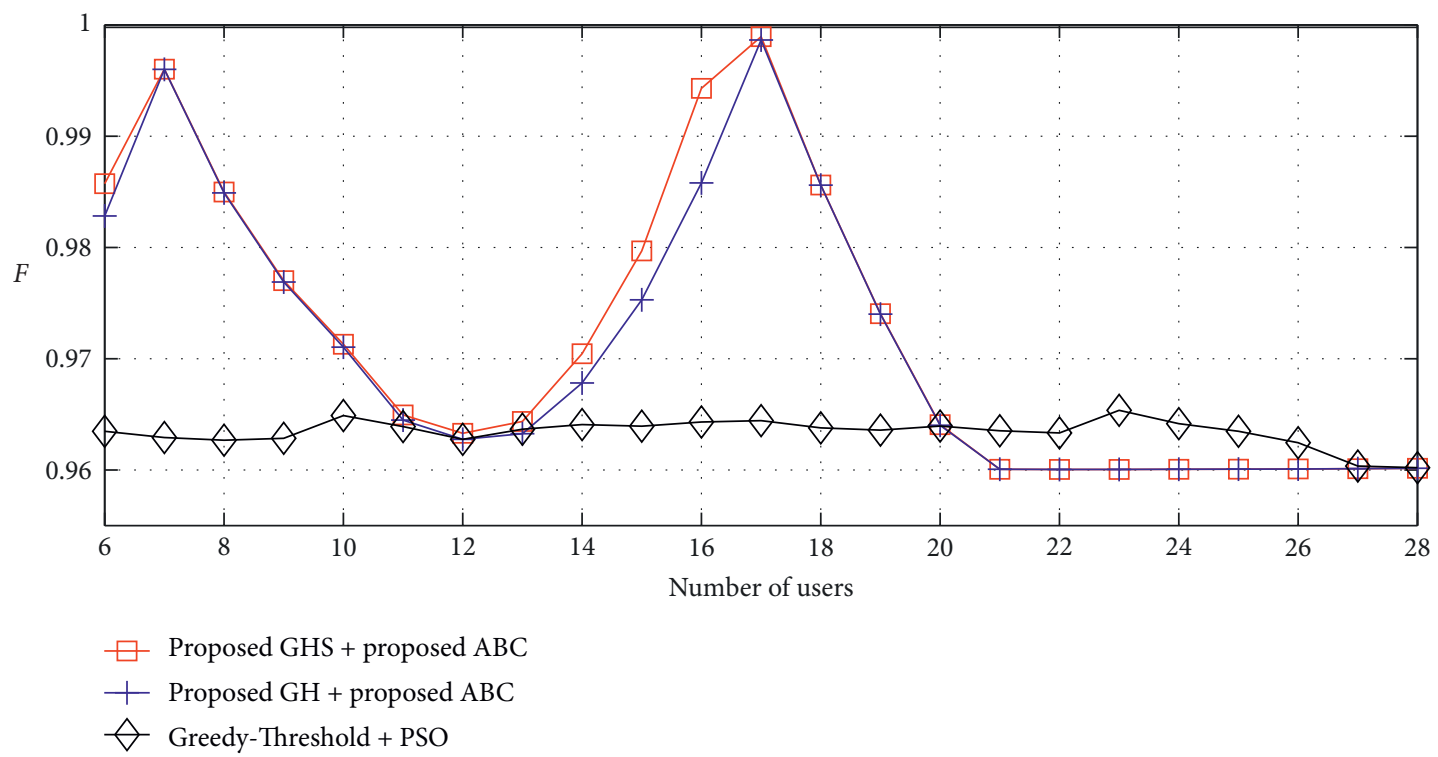

(b)

FIGURE 10: Results obtained by the proposed GH/GHS combined with the proposed ABC and Greedy-Threshold combined with PSO $[17,18]$ in ensuring the required level of proportional fairness $(0.96)$ as the data rate proportionality is $16: 1: \cdots: 1$. (a) Obtained sum data rate. (b) Obtained fairness.

Note that our proposed hybrid OFDMA resource allocation scheme is a suboptimal method for ensuring required level of proportional fairness and obtains near-optimal solutions for the rate adaptive resource allocation problem (1)-(4). Because of the NP-hard nature of the problem (1)-(4), it is hard to obtain its optimal solution and, therefore, difficult to estimate the gap between our solutions and the optimal solution.

Besides, as can be seen from the Figures, curves of sum data rates and fairness are not smooth and fluctuate because sum data rates and fairness obtained by the proposed GH/ GHS are easily influenced by the optimal number of the remaining subcarriers, i.e., $N_{\mathrm{op}}$. It can be seen from
Figures 2-4 that $N_{\text {op }}$ varies with the number of users. Additionally, it can be obviously observed from Figures 5 and 6 together with Figures 2-4 that as $N_{\text {op }}$ tends to be zero, fairness becomes larger while sum data rate becomes smaller, thereby causing curves to be nonsmooth.

\section{Conclusions}

In this paper, a hybrid OFDMA resource allocation scheme is presented by first using the proposed combination of Hungarian algorithm with the greedy method (i.e., the proposed GH/GHS) for subcarrier allocation and then using the proposed $\mathrm{ABC}$ for power allocation. For one thing, the 
TABLE 2: Results obtained for a $K=28$ instance by PSO and proposed ABC at different parameters in 100 runs based on subcarrier Allocation of GHS as the data rate proportionality is $16: 1: \cdots: 1$.

\begin{tabular}{|c|c|c|c|c|c|c|c|c|c|c|c|}
\hline \multirow{2}{*}{ Algorithms } & \multirow{2}{*}{\multicolumn{2}{|c|}{ Parameters }} & \multicolumn{4}{|c|}{ Sum data rate with $F \geq 0.96$} & \multicolumn{5}{|c|}{ Fairness $(F)$} \\
\hline & & & $\operatorname{Max} . \times 10^{7}$ & Min. $\times 10^{7}$ & Mean $\times 10^{7}$ & Std $\times 10^{3}$ & Max. & Min. & Mean & Std $\times 10^{-4}$ & $N_{F} \geq 0.96$ \\
\hline \multirow{5}{*}{ Proposed GHS + PSO $[17,18]$} & \multirow{5}{*}{$V_{\max }$} & $1 \times 10^{-4}$ & 1.3951 & 0.9952 & 1.2737 & 733.21 & 0.9633 & 0.9600 & 0.9607 & 6.5183 & 100 \\
\hline & & $2 \times 10^{-4}$ & 1.4237 & 1.0690 & 1.2766 & 745.63 & 0.9637 & 0.9563 & 0.9605 & 8.7357 & 97 \\
\hline & & $3 \times 10^{-4}$ & 1.4419 & 1.0947 & 1.2859 & 689.21 & 0.9632 & 0.9553 & 0.9607 & 11.276 & 87 \\
\hline & & $4 \times 10^{-4}$ & 1.4142 & 1.1314 & 1.2900 & 707.61 & 0.9649 & 0.9520 & 0.9609 & 23.659 & 70 \\
\hline & & 0.001 & 1.3979 & 1.2061 & 1.2972 & 484.01 & 0.9616 & 0.9488 & 0.9605 & 31.745 & 17 \\
\hline \multirow{10}{*}{ Proposed GHS + proposed ABC } & \multirow{6}{*}{$f_{1}(\mathrm{SPP}=1000)$} & 0.96 & 1.4848 & 1.4830 & 1.4839 & 3.8343 & 0.9602 & 0.9600 & 0.9601 & 0.5755 & 100 \\
\hline & & 10 & 1.4844 & 1.4830 & 1.4839 & 3.1775 & 0.9604 & 0.9600 & 0.9601 & 1.1954 & 100 \\
\hline & & 100 & 1.4849 & 1.4832 & 1.4837 & 3.3902 & 0.9603 & 0.9600 & 0.9601 & 0.5088 & 100 \\
\hline & & 250 & 1.4844 & 1.4831 & 1.4839 & 4.1048 & 0.9602 & 0.9600 & 0.9601 & 0.5294 & 100 \\
\hline & & 500 & 1.4850 & 1.4830 & 1.4838 & 5.4311 & 0.9603 & 0.9600 & 0.9601 & 0.7555 & 100 \\
\hline & & 5000 & 1.4850 & 1.4833 & 1.4839 & 3.6674 & 0.9602 & 0.9600 & 0.9601 & 0.4694 & 100 \\
\hline & \multirow{4}{*}{$\operatorname{SPP}\left(f_{1}=5000\right)$} & 10 & 1.4845 & 1.4812 & 1.4829 & 6.7544 & 0.9604 & 0.9600 & 0.9601 & 1.0246 & 100 \\
\hline & & 50 & 1.4847 & 1.4831 & 1.4838 & 3.5313 & 0.9603 & 0.9600 & 0.9601 & 0.7345 & 100 \\
\hline & & 500 & 1.4848 & 1.4828 & 1.4838 & 3.9949 & 0.9603 & 0.9600 & 0.9601 & 0.6177 & 100 \\
\hline & & 1000 & 1.4850 & 1.4833 & 1.4839 & 3.6674 & 0.9602 & 0.9600 & 0.9601 & 0.4694 & 100 \\
\hline
\end{tabular}

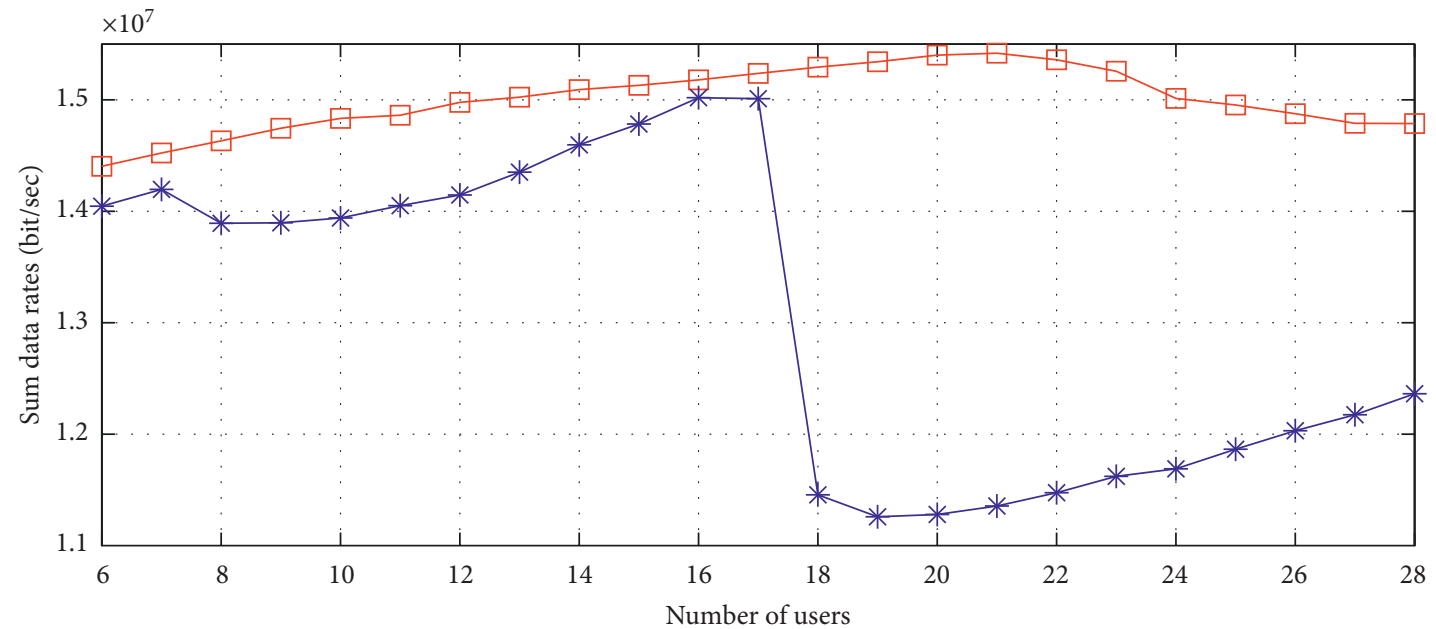

$\square$ Proposed GHS + proposed ABC

$*$ Proposed GHS with strict fairness [5]

(a)

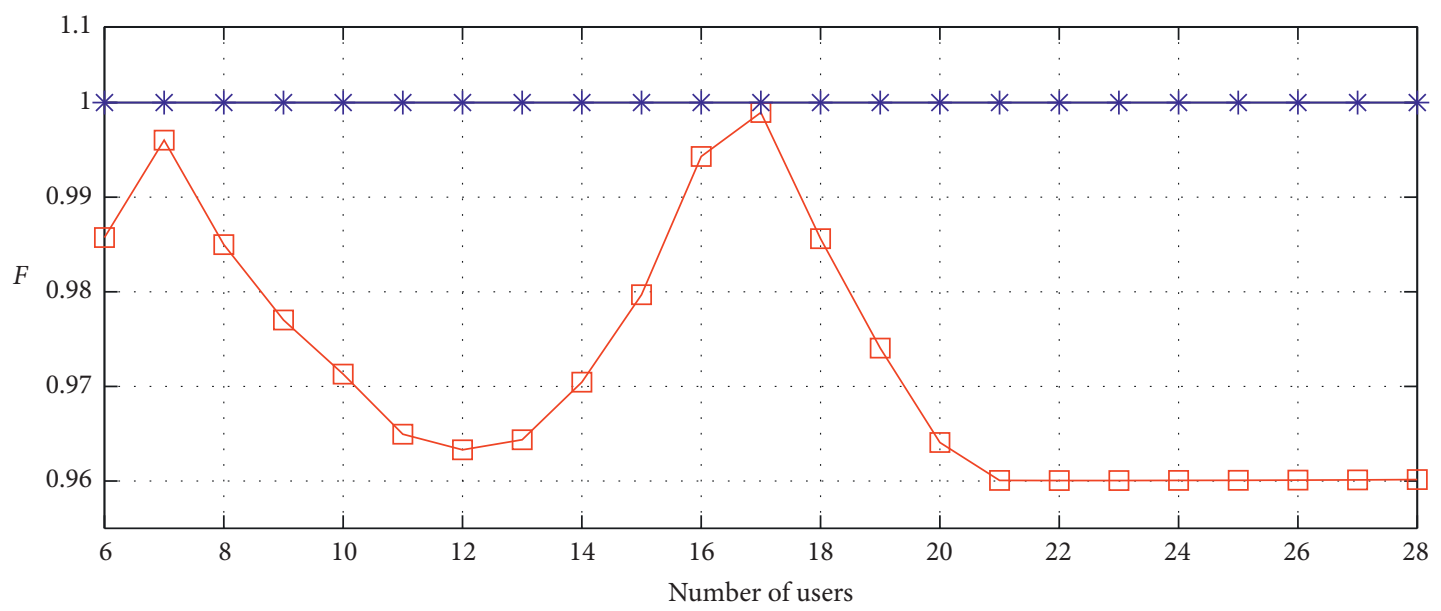

Proposed GHS + proposed ABC

$*$ Proposed GHS with strict fairness [5]

(b)

FIGURE 11: Comparison results between the proposed ABC-based power allocation and the power allocation with strict fairness [5] based on subcarrier allocation obtained by the proposed GHS. (a) Sum data rates. (b) Fairness. 
TABLE 3: Complexity comparisons among different algorithms.

\begin{tabular}{|c|c|c|}
\hline \multirow{2}{*}{ Algorithms } & \multicolumn{2}{|c|}{ Complexity } \\
\hline & Subcarrier allocation & Power allocation \\
\hline Proposed GH & $O\left(K N \log _{2} N\right)$ if $K N \log _{2} N \geq K^{3} ;$ otherwise, $O\left(K^{3}\right)$ & $O(K)$ \\
\hline Proposed GHS & $\mathrm{O}\left(K^{5}\right)$ & $O(K)$ \\
\hline Proposed $\mathrm{GH}+$ proposed $\mathrm{ABC}$ & $O\left(K N \log _{2} N\right)$ if $K N \log _{2} N \geq K^{3} ;$ otherwise, $O\left(K^{3}\right)$ & $O(2 * N * S *$ MaxCycle $)$ \\
\hline Proposed GHS + proposed ABC & $O\left(K^{5}\right)$ & $O(2 * N * S *$ MaxCycle $)$ \\
\hline Ant-CNS [14] & $O(K * N * S *$ MaxCycle $)$ & $\mathrm{O}(\mathrm{K})$ \\
\hline ABC-OSA [15] & $O(K * N * S *$ MaxCycle $)$ & $\mathrm{O}(\mathrm{K})$ \\
\hline Greedy-EP [19] & $O\left(K N \log _{2} N\right)$ & $\mathrm{O}(\mathrm{K})$ \\
\hline Greedy-LP [19] & $O\left(K N \log _{2} N\right)$ & $\mathrm{O}(\mathrm{K})$ \\
\hline Greedy-Threshold + PSO $[17,18]$ & $O\left(K N \log _{2} N\right)$ & $O(N * S *$ MaxCycle $)$ \\
\hline
\end{tabular}

proposed GH/GHS can make full use of the advantages of both the globally optimal Hungarian algorithm in improving sum data rates and the locally optimal greedy method in holding the acceptable proportional fairness. Compared with GH, GHS can make Hungarian algorithm work in the searching mode to further improve sum data rates and fairness. For another, if the fairness obtained by the proposed GH/GHS in subcarrier allocation is lower than the required level of proportional fairness, the proposed $\mathrm{ABC}$ based power allocation can be used to ensure the required level of proportional fairness and maximize the sum data rates.

Simulation results show the following: (1) The proposed GH/GHS can obtain the acceptable proportional fairness while there are higher sum data rates than greedy methods and evolutionary algorithms even if subcarriers are relatively insufficient in allocation to users, and the proposed GHS is obviously superior to the proposed GH as $N^{*}$ is equal or close to zero. (2) As the fairness obtained by the proposed GH/GHS in subcarrier allocation cannot achieve the required level of proportional fairness, the proposed ABC-based power allocation can effectively ensure the required level of proportional fairness and obtain higher sum data rates than PSObased power allocation. (3) The proposed ABC-based power allocation is less affected by parameters of $f_{1}$ and SPP but could obtain higher sum data rates with larger $f_{1}$ or SPP, thereby performing more steadily than the PSObased power allocation.

Complexity analysis shows that the proposed $\mathrm{GH}$ has complexity of $\mathrm{O}\left(\mathrm{KN} \log _{2} \mathrm{~N}\right)$ or $\mathrm{O}\left(\mathrm{K}^{3}\right)$, the proposed GHS has complexity of $O\left(K^{5}\right)$, and the proposed ABC-based power allocation has complexity of $O(2 * N * S *$ MaxCycle $)$. Complexity comparisons indicate that complexity of the proposed GH/GHS may lie between that of greedy methods and that of evolutionary algorithms, and complexity of the proposed $\mathrm{ABC}$-based power allocation is slightly higher than that of PSO-based power allocation.

\section{Data Availability}

The data used in the experiments are generated randomly with Rayleigh distribution. All the data included in this study are available from the corresponding author (Ming Sun, e-mail: snogisunming@163.com) upon request.

\section{Conflicts of Interest}

The authors declare no conflicts of interest.

\section{Acknowledgments}

This work was supported in part by the National Natural Science Foundation of China under Grants 61872204 and 71803095, the Joint guiding project of Natural Science Foundation of Heilongjiang Province under Grant LH2019F038, the Young Innovative Talents Program of Basic Business Special Project of Heilongjiang Provincial Education Department under Grant 135309340, and the Science and Technology Project of Qiqihar under Grant GYGG-201915.

\section{References}

[1] Z. E. Ankarali, B. Peköz, and H. Arslan, "Enhanced OFDM for 5G RAN," ZTE Communications, vol. 15, no. s1, pp. 11-20, 2017.

[2] J. Ghosh, D. N. K. Qaraqe, and M. Qaraqeb, "Downlink capacity of OFDMA-CR based 5G femtocell networks," Physical Communication, vol. 29, pp. 329-335, 2018.

[3] S. Niknam, A. A. Nasir, H. Mehrpouyan, and B. Natarajan, "A multiband OFDMA heterogeneous network for millimeter wave 5G wireless applications," IEEE Access, vol. 4, pp. 5640-5648, 2016.

[4] P. Wei, L. Dan, Y. Xiao, W. Xiang, and S. Li, "Improved Ncontinuous OFDM for 5G wireless communications," 2016, https://arxiv.org/abs/1601.04795.

[5] Z. Yin, S. Zhuang, Z. Wu, and B. Ma, "Rate adaptive based resource allocation with proportional fairness constraints in OFDMA systems," Sensors, vol. 15, no. 10, pp. 24996-25014, 2015.

[6] M. Sun, K. Y. Lee, Y. Xu, and W. Bai, "Hysteretic noisy chaotic neural networks for resource allocation in OFDMA system," IEEE Transactions on Neural Networks and Learning Systems, vol. 29, no. 2, pp. 273-285, 2018.

[7] H. B. Zhang and X. X. Wang, "Resource allocation for downlink OFDM system using noisy chaotic neural network," Electronics Letters, vol. 47, no. 21, pp. 1201-1202, 2011.

[8] J. Yuan, F. Zhang, J. Wang, Y. Wang, J. Lin, and Y. Pang, "OFDMA adaptive resource allocation based on fairness and penalty function," Systems Engineering and Electronics, vol. 40, no. 2, pp. 427-434, 2018.

[9] H. Zhang, X. Wang, H. Dai, and F. Li, "Channel-aware adaptive resource allocation for multicast and unicast services 
in orthogonal frequency division multiplexing systems," IET Communications, vol. 6, no. 17, pp. 3006-3014, 2012.

[10] C. K. Tan, T. C. Chuah, and S. W. Tan, "A coalitional gamebased algorithm for OFDMA resource allocation in multicast cognitive radio networks," Wireless Personal Communications, vol. 80, no. 1, pp. 415-427, 2015.

[11] C. Liao, J. Wu, J. Du, and L. Zhao, "Ant colony optimization inspired resource allocation for multiuser multicarrier systems," in Proceedings of the 2017 9th International Conference on Wireless Communications and Signal Processing (WCSP), Nanjing, China, 2017.

[12] L. Jiang and R. Song, "A low-complexity resource allocation scheme for OFDMA multicast systems with proportional fairness," China Communications, vol. 15, no. 1, pp. 1-11, 2018.

[13] Z. Shen, J. Andrews, and B. L. Evans, "Adaptive resource allocation in multiuser OFDM systems with proportional rate constraints," IEEE Transactions on Wireless Communications, vol. 4, no. 6, pp. 2726-2737, 2005.

[14] F. Wang, X. Liao, S. Guo, and H. Huang, "Joint subcarrier and power allocation with fairness in uplink OFDMA systems based on ant colony optimization," International Journal of Communication Systems, vol. 27, no. 10, pp. 1505-1521, 2014.

[15] N. Sharma and A. Anpalagan, "Bee colony optimization aided adaptive resource allocation in OFDMA systems with proportional rate constraints," Wireless Networks, vol. 20, no. 7, pp. 1699-1713, 2014.

[16] N. Sharma and A. S. Madhukumar, "Genetic algorithm aided proportional fair resource allocation in multicast OFDM systems," IEEE Transactions on Broadcasting, vol. 61, no. 1, pp. 16-29, 2015.

[17] H. Liang, X. Zhao, and C. Zhang, "Fair adaptive resource allocation in NC-OFDM based cognitive radio system," in Proceedings of the 2010 International Conference on Wireless Communications \& Signal Processing (WCSP), Suzhou, China, 2010.

[18] C. Zhang and X. Zhao, "Adaptive resource allocation in multiuser OFDM system based on fairness threshold," Journal of Communication, vol. 32, no. 12, pp. 65-71, 2011.

[19] I. C. Wong, Z. Shen, B. L. Evants, and J. G. Andrews, “A low complexity algorithm for proportional resource allocation in OFDMA systems," in Proceedings of the 2004 IEEE Workshop on Signal Processing Systems, pp. 1-6, Austin, TX, USA, 2004.

[20] M. Ashourian, R. Salimian, and H. Mahdavi-Nasab, "A low complexity resource allocation method for OFDMA system based on channel gain," Wireless Personal Communications, vol. 71, no. 1, pp. 519-529, 2013.

[21] N. Forouzan and S. A. Ghorashi, "New algorithm for joint subchannel and power allocation in multi-cell OFDMA-based cognitive radio networks," IET Communications, vol. 8, no. 4, pp. 508-515, 2014.

[22] S. Akhlaghi, I. Hajari, and E. Rahimi, "Resource allocation for downlink of multi-user multi-band wireless networks," Electronics Letters, vol. 47, no. 3, pp. 220-221, 2011.

[23] H. Zhang, X. Peng, and S. Chen, "Resource allocation based on femto base station in Macrocell-Femtocell networks," Journal of Computer Applications, vol. 37, no. 5, pp. 13111316, 2017.

[24] D. Karaboga and B. Akay, "A modified artificial bee colony (ABC) algorithm for constrained optimization problems," Applied Soft Computing, vol. 11, no. 3, pp. 3021-3031, 2011.

[25] K. Deb, "An efficient constraint handling method for genetic algorithms," Computer Methods in Applied Mechanics and Engineering, vol. 186, no. 2-4, pp. 311-338, 2000.
[26] J. Yuan, J. Wang, P. Qiu, Y. Wang, J. Lin, and Y. Pang, "Adaptive resource allocation based on artificial bee colony algorithm and simulated annealing algorithm for multiuser OFDM systems," International Journal of Science, vol. 3, no. 11, pp. 113-125, 2016.

[27] J. Yuan, J. Wang, F. Zhang, Y. Wang, J. Lin, and Y. Pang, "Adaptive resource allocation for multiuser OFDM based on artificial bee colony algorithm," Journal of Jilin University (Engineering and Technology Edition), vol. 49, no. 2, pp. 624-630, 2019.

[28] Y. Yang, Q. Zhang, Y. Wang, T. Emoto, M. Akutagawa, and S. Konaka, "Adaptive resources allocation algorithm based on modified PSO for cognitive radio system," China Communications, vol. 16, no. 5, pp. 83-92, 2019.

[29] Z. Yang, W. Xu, and Y. Li, "Fair non-orthogonal multiple access for visible light communication downlinks," IEEE Wireless Communications Letters, vol. 6, no. 1, pp. 66-69, 2017.

[30] Z. Yang, Mi. Chen, W. Saad, W. Xu, and M. Shikh-Bahaei, "Sum-rate maximization of uplink rate splitting multiple access (RSMA) communication," 2019, https://arxiv.org/abs/ 1906.04092.

[31] M. Chen, Z. Yang, W. Saad, C. Yin, H. Vincent Poor, and S. Cui, "A joint learning and communications framework for federated learning over wireless networks," 2020, https:// arxiv.org/abs/1909.07972. 